This is the final peer-reviewed accepted manuscript of:

Meng-Kiat Chuah, Rita Fioresi, Equal rank real forms of affine non-twisted KacMoody Lie superalgebras, Journal of Pure and Applied Algebra, Volume 224, Issue 7, 2020, 106278, ISSN 0022-4049

The final published version is available online at:

https://doi.org/10.1016/j.jpaa.2019.106278

Rights / License:

The terms and conditions for the reuse of this version of the manuscript are specified in the publishing policy. For all terms of use and more information see the publisher's website.

This item was downloaded from IRIS Università di Bologna (https://cris.unibo.it/)

When citing, please refer to the published version. 


\title{
EQUAL RANK REAL FORMS OF AFFINE NON-TWISTED KaC-MoOdy Lie SUPERALGeBras
}

\author{
Meng-Kiat Chuah \\ Department of Mathematics, National Tsing Hua University, \\ Hsinchu 300, Taiwan. \\ chuah@math.nthu.edu.tw \\ Rita Fioresi \\ Dipartimento di Matematica, University of Bologna, \\ Piazza Porta San Donato 5, 40126 Bologna, Italy. \\ rita.fioresi@unibo.it
}

\begin{abstract}
:
We study the equal rank real forms of affine non-twisted Kac-Moody Lie superalgebras by Cartan automorphisms and Vogan diagrams. We introduce admissible positive systems and Hermitian real forms, then show that a real form has admissible positive system if and only if it is Hermitian. As a result, we use the Vogan diagrams to classify the Hermitian real forms.
\end{abstract}

\section{Mathematics Subject Classification:}

17B22, 17B67.

\section{Keywords:}

affine Kac-Moody Lie superalgebras, equal rank real forms, Vogan diagrams, Cartan automorphisms, admissible positive systems, Hermitian real forms. 


\section{Introduction}

Let $\stackrel{\circ}{L}$ be a finite dimensional complex contragredient Lie superalgebra [15]. Let $L$ be its affinization, namely the corresponding affine non-twisted Kac-Moody superalgebra. We let subscripts $\overline{0}$ and $\overline{1}$ denote the even and odd parts, so for example $L=L_{\overline{0}}+L_{\overline{1}}$. Let $\mathfrak{g}$ be a real form of $L$, namely a real subalgebra such that $L=\mathfrak{g} \oplus i \mathfrak{g}$. Let $\mathfrak{k}$ be a maximal compact subalgebra of $\mathfrak{g}_{\overline{0}}$, namely $\mathfrak{k}$ is the fixed points of a Cartan involution on $\mathfrak{g}_{\overline{0}}[2$, Sec. 3]. A Cartan decomposition of $\mathfrak{g}$ is

$$
\mathfrak{g}=\mathfrak{k}+\mathfrak{p},
$$

where $\mathfrak{p}$ is the orthogonal complement of $\mathfrak{k}$ with respect to an invariant non-degenerate supersymmetric bilinear form. All lower case Gothic letters denote real subalgebras or real subspaces, with subscript $\mathbb{C}$ for their complexification, complex subalgebras or complex subspaces. So for example, $L=\mathfrak{k}_{\mathbb{C}}+\mathfrak{p}_{\mathbb{C}}$. Let $\mathfrak{h}$ be a Cartan subalgebra of $\mathfrak{g}$. We have the root space decomposition $L=\mathfrak{h}_{\mathbb{C}}+\sum_{\Delta} L_{\alpha}$. We say that a positive system $\Delta^{+}$is admissible if

$$
\left[\mathfrak{k}_{\mathbb{C}}, \mathfrak{p}_{\mathbb{C}}^{ \pm}\right] \subset \mathfrak{p}_{\mathbb{C}}^{ \pm},\left[\mathfrak{p}_{\mathbb{C}}^{ \pm}, \mathfrak{p}_{\mathbb{C}}^{ \pm}\right]=0 .
$$

where $\mathfrak{p}_{\mathbb{C}}^{ \pm}=\mathfrak{p}_{\mathbb{C}} \cap \sum_{\Delta^{ \pm}} L_{\alpha}$. In general $\mathfrak{p}_{\mathbb{C}} \supset \mathfrak{p}_{\mathbb{C}}^{+}+\mathfrak{p}_{\mathbb{C}}^{-}$, with equality holding when $\mathfrak{h} \subset \mathfrak{k}$, that is, when $\mathfrak{h}$ is a compact Cartan subalgebra. In this case, we say that $\mathfrak{g}$ satisfies the equal rank condition because $\operatorname{rank}(\mathfrak{g})=\operatorname{rank}(\mathfrak{k})$.

When $\mathfrak{g}=\mathfrak{g}_{\overline{0}}$, the equal rank condition is a necessary, though not sufficient, condition for the existence of admissible positive systems [9, 10]. So we assume it from now on. The real forms leading to admissible positive systems are called Hermitian, and they are especially important when studying representation theory. They are classically linked to the existence of natural invariant complex structures on the symmetric spaces $G / K$, where $G$ is a real group with Lie algebra $\mathfrak{g}$, and $K$ is its maximal compact subgroup. When $\mathfrak{g}$ is finite dimensional, the infinite dimensional representations of $G$ can be realized within the space of sections of a suitable complex vector bundle on $G / K$ (Refer to [14] for the classical theory, to [1, 3, 13, 4] for its super extension). Hence, there are potential important applications for the representation theory of affine superalgebras and supergroups.

Let $\operatorname{aut}_{2,4}(L)$ denote the $L$-automorphisms of order 2 on $L_{\overline{0}}$, and order 4 on $L_{\overline{1}}$. As in the finite dimensional case, a key role in the classification of the real forms is played by aut ${ }_{2,4}(L)$ and the Vogan diagrams. We say that $\theta \in \operatorname{aut}_{2,4}(L)$ is equal rank if $\operatorname{rank}\left(L^{\theta}\right)=\operatorname{rank}(L)$. We call $\theta$ a Cartan automorphism of $\mathfrak{g}$ if it satisfies Definition 2.1(c). This generalizes the Cartan involutions of real semisimple Lie algebras.

Let $\mathrm{D}$ be the Dynkin diagram of $L$ [5, Fig. 1]. We call a Vogan diagram on $\mathrm{D}$ a function $c$ which assigns \pm 1 to white and $\pm i$ to dark (i.e. grey or black) vertices. 
When we draw a Vogan diagram, we make the convention to circle the white and dark vertices assigned with -1 or $-i$. We say that a Vogan diagram on D represents $\theta \in \operatorname{aut}_{2,4}(L)$ if (3.5) is satisfied. We now state the main results of our paper.

Theorem 1.1. Let $L$ be an affine non-twisted Kac-Moody Lie superalgebra. Up to equivalence, there is a bijective correspondence among the following:

(a) Equal rank real forms $\mathfrak{g}$ of $L$,

(b) Equal rank $\theta \in \operatorname{aut}_{2,4}(L)$,

(c) Vogan diagrams $c$ on $\mathrm{D}$.

In this correspondence, $\theta$ is a Cartan automorphism of $\mathfrak{g}$, and c represents $\theta$.

In Theorem 1.1, the notions of equivalent real forms $\mathfrak{g}, \mathfrak{g}^{\prime}$ and equivalent automorphisms $\theta, \theta^{\prime}$ are given by $\sigma(\mathfrak{g})=\mathfrak{g}^{\prime}$ and $\sigma \theta \sigma^{-1}=\theta^{\prime}$ for some inner automorphism $\sigma$ on $L$. The notion of equivalent Vogan diagrams is given in Definition 3.1.

By Theorem 1.1, we can use the Vogan diagrams to classify the equal rank real forms. Let the imaginary roots be $\{n \delta \subset \Delta ; 0 \neq n \in \mathbb{Z}\}$. We say that $\delta$ is compact (resp. non-compact) if $L_{\delta} \subset \mathfrak{k}_{\mathbb{C}}\left(\right.$ resp. $\left.L_{\delta} \subset \mathfrak{p}_{\mathbb{C}}\right)$.

Corollary 1.2. Up to equivalence, an equal rank real form $\mathfrak{s}$ of $\stackrel{\circ}{L}$ has exactly two extensions to real forms $\widehat{\mathfrak{s}}_{+}$and $\widehat{\mathfrak{s}}_{-}$of $L$, for $\delta$ compact and non-compact respectively. Conversely, every equal rank real form of $L$ can be expressed as $\widehat{\mathfrak{s}}_{ \pm}$. For all equal rank real forms $\mathfrak{s}, \mathfrak{s}^{\prime}$ of $\stackrel{\circ}{L}$,

(a) $\widehat{\mathfrak{s}}_{+} \cong \widehat{\mathfrak{s}}_{+}^{\prime} \Longleftrightarrow \mathfrak{s} \cong \mathfrak{s}^{\prime}$

(b) $\widehat{\mathfrak{s}}_{-} \cong \widehat{\mathfrak{s}}_{-}^{\prime}$.

We now turn to the Hermitian real forms.

Definition 1.3. A real form $\mathfrak{g}$ of $L$ is said to be Hermitian if $\mathfrak{g} \cap \stackrel{\circ}{L}$ is Hermitian and $\delta$ is compact.

Theorem 1.4. There exists an admissible positive system for a real form $\mathfrak{g}$ if and only if $\mathfrak{g}$ is Hermitian.

Theorem 1.5. All Hermitian real forms of $L$ are represented by the Vogan diagrams in Figure 1.

Figure 1 represents all Hermitian real forms of $L$. By Corollary 1.2, they are denoted $\widehat{\mathfrak{s}}_{+}$with the following $\mathfrak{s}_{\overline{0}}$. Let $\operatorname{rank}(\stackrel{\circ}{L})=n=p+q$ for all classical superalgebras.

$$
\begin{aligned}
& \mathfrak{s u}(r, p-r)+\mathfrak{s u}(s, q+1-s)+i \mathbb{R} \\
& \mathfrak{s p}(n-1)+i \mathbb{R} \quad \mathfrak{s p}(n-1, \mathbb{R})+i \mathbb{R} \\
& \mathfrak{s p}(n, \mathbb{R}) \quad \mathfrak{s o}(2 p+1)+\mathfrak{s p}(q, \mathbb{R}) \\
& \mathfrak{s o}(2 p-1,2)+\mathfrak{s p}(q, \mathbb{R}) \quad \mathfrak{s o}^{*}(2 p)+\mathfrak{s p}(q) \\
& \mathfrak{s o}(2 p)+\mathfrak{s p}(q, \mathbb{R}) \quad \mathfrak{s o}(2 p-2,2)+\mathfrak{s p}(q, \mathbb{R}) \\
& \mathfrak{s l}(2, \mathbb{R})^{3} \quad \mathfrak{s u}(2)^{2}+\mathfrak{s l}(2, \mathbb{R}) \quad \mathfrak{s l}(2, \mathbb{R})+\mathfrak{s o}(7) \quad \mathfrak{s u}(2)+\mathfrak{s o}(5,2)
\end{aligned}
$$



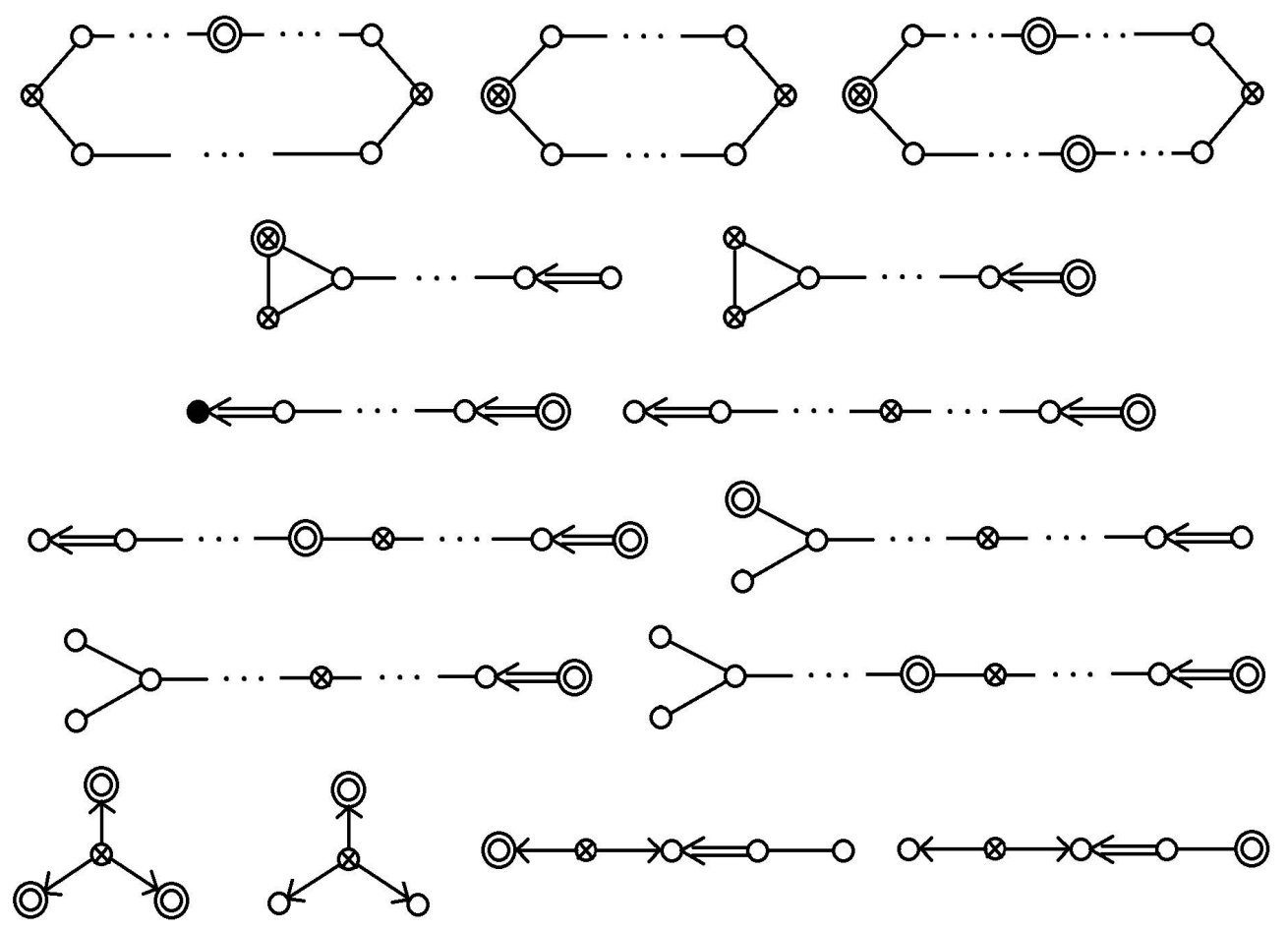

Figure 1: Hermitian real forms of $L$.

The sections in this article are arranged as follows. In Section 2, we study the correspondence between real forms and Cartan automorphisms. In Section 3, we study the correspondence between Cartan automorphisms and Vogan diagrams, and prove Theorem 1.1 and Corollary 1.2. In Section 4, we prove Theorem 1.4, which establishes the equivalence between Hermitian real forms and admissible positive systems. We then prove Theorem 1.5, which classifies the Hermitian real forms by Vogan diagrams. In Theorem 4.1 we also discuss the related Iwasawa decomposition and complex structure. In Section 5, we provide some examples to illustrate the Dynkin and Vogan diagrams, together with their related concepts.

We have added an appendix in Section 6. It applies Kumar's work [18] to prove that inner automorphisms on $L_{\overline{0}}$ can be extended to automorphisms on $L$ (Theorem 6.1). This result is used in earlier arguments such as Proposition 2.4 and Theorem 3.3. Its proof has a different flavor than the rest of this article, so we treat it separately in Section 6. 


\section{Real forms and Cartan automorphisms}

Let $L$ be an affine non-twisted Lie superalgebra. In this section we establish a bijective correspondence between the two items in Theorem 1.1(a,b), namely the equivalence classes of equal rank real forms of $L$ and equal rank automorphisms in $\operatorname{aut}_{2,4}(L)$.

We briefly recall the non-super setting. The real forms of $L_{\overline{0}}$ correspond to $\mathbb{C}$ linear automorphisms $\theta_{\overline{0}}: L_{\overline{0}} \longrightarrow L_{\overline{0}}$ such that $\theta_{\overline{0}}^{2}=1$ [2, p. 217]. They are called involutions and are denoted by $\operatorname{inv}\left(L_{\overline{0}}\right)$. We define involutions of the first and second kinds by the existence of a Cartan subalgebra $\mathfrak{h}_{\mathbb{C}}$, a Borel subalgebra $\mathfrak{b}_{\mathbb{C}}$ and its opposite Borel subalgebra $\mathfrak{b}_{\mathbb{C}}^{-}$such that

$$
\begin{aligned}
& \text { (a) } \theta_{\overline{0}} \text { first kind } \Longleftrightarrow \theta_{\overline{0}}\left(\mathfrak{h}_{\mathbb{C}}\right)=\mathfrak{h}_{\mathbb{C}}, \theta_{\overline{0}}\left(\mathfrak{b}_{\mathbb{C}}\right)=\mathfrak{b}_{\mathbb{C}} \\
& \text { (b) } \theta_{\overline{0}} \text { second kind } \Longleftrightarrow \theta_{\overline{0}}\left(\mathfrak{h}_{\mathbb{C}}\right)=\mathfrak{h}_{\mathbb{C}}, \theta_{\overline{0}}\left(\mathfrak{b}_{\mathbb{C}}\right)=\mathfrak{b}_{\mathbb{C}}^{-} \text {. }
\end{aligned}
$$

Every $\theta_{\overline{0}} \in \operatorname{inv}\left(L_{\overline{0}}\right)$ is of either the first kind or the second kind, but not both [19, Part III] $[2,(3.1)]$. We shall focus on the involutions of the first kind. We define the almost compact real forms by $[2,(3.7)]$

$\left\{\right.$ almost compact real forms $\mathfrak{g}_{\overline{0}}$ of $\left.L_{\overline{0}}\right\} \longleftrightarrow\left\{\theta_{\overline{0}} \in \operatorname{inv}\left(L_{\overline{0}}\right)\right.$ of the first kind $\}$

where $\theta_{\overline{0}}$ stabilizes $\mathfrak{g}_{\overline{0}}$, and $\theta_{\overline{0}}$ is a Cartan involution of $\mathfrak{g}_{\overline{0}}$. The Cartan decomposition of $\mathfrak{g}_{\overline{0}}$ is $\mathfrak{g}_{\overline{0}}=\mathfrak{k}_{\overline{0}}+\mathfrak{p}_{\overline{0}}$, where $\theta_{\overline{0}}$ has eigenvalues 1 and -1 on $\mathfrak{k}_{\overline{0}}$ and $\mathfrak{p}_{\overline{0}}$ respectively.

We now return to the super setting. Let $L$ be the non-twisted affinization of a contragredient Lie superalgebra $\stackrel{\circ}{L}$. It has an invariant non-degenerate supersymmetric bilinear form $B$, which is unique up to scalar. For a given $\theta \in \operatorname{aut}_{2,4}(L)$, let $L^{\theta}$ denote the elements of $L$ which are fixed by $\theta$. Let $B_{\theta}(x, y)=B(x, \theta(y))$.

\section{Definition 2.1.}

(a) We say that a real form $\mathfrak{g}$ of $L$ is of equal rank if $\mathfrak{g}=\stackrel{\circ}{L} \cap \mathfrak{g}$ is a real form of $\stackrel{\circ}{L}$, and the Cartan decomposition $\mathfrak{\mathfrak { g }}=\mathfrak{k} \oplus \mathfrak{\mathfrak { p }}$ satisfies $\operatorname{rank}(\mathfrak{k})=\operatorname{rank}(\mathfrak{g})$.

(b) We say that $\theta \in \operatorname{aut}_{2,4}(L)$ is of equal rank if $\operatorname{rank}(L)=\operatorname{rank}\left(L^{\theta}\right)$.

(c) We say that $\theta \in \operatorname{aut}_{2,4}(L)$ is a Cartan automorphism of a real form $\mathfrak{g}$ of $L$ if $\theta(\mathfrak{g})=\mathfrak{g}$, and $-B_{\theta}$ is an inner product on $\mathfrak{g}$.

Let $\mathfrak{g}$ be an equal rank real form of $L$ and let $\mathfrak{h}$ be a compact (i.e. $\mathfrak{h} \subset \mathfrak{k}$ ) Cartan subalgebra of $\mathfrak{g}_{\overline{0}}$. All such $\mathfrak{h}$ are conjugate in $L_{\overline{0}}$ under the inner group of $L_{\overline{0}}[2, \mathrm{p}$. 227], hence by Theorem 6.1 also conjugate by the inner group of $L$. Let $\Delta$ be the root system, with root space decomposition $L=\mathfrak{h}_{\mathbb{C}}+\sum_{\Delta} L_{\alpha}$. We shall denote a general nonzero root vector by $x_{\alpha} \in L_{\alpha}$. Let $\stackrel{\circ}{\Delta}$ be the roots of $\stackrel{\circ}{L}$, and let $\delta$ be a generator of the imaginary roots. We have $[21, \S 18.2]$

$$
\Delta=\{\alpha+n \delta ; \alpha \in \stackrel{\Delta}{\Delta} \cup\{0\}, n \in \mathbb{Z}\} \backslash\{0\} .
$$


Let $\theta$ be a Cartan automorphism of $\mathfrak{g}$. For $\mathfrak{g}$ of equal rank, $\theta$ stabilizes some $\mathfrak{g}$. We let $\stackrel{\circ}{\theta}$ and $\theta_{\overline{0}}$ be the restrictions of $\theta$ to $\mathfrak{g}$ and $\mathfrak{g}_{\overline{0}}$ respectively.

Proposition 2.2. Let $\theta \in \operatorname{aut}_{2,4}(L)$ be of equal rank. Then there exists an equal rank real form $\mathfrak{g}$ of $L$, with $\theta$ as its Cartan automorphism.

Proof. Given such a $\theta$, there exists a real form $\mathfrak{g}_{\overline{0}}$ of $L_{\overline{0}}$ with $\theta_{0}$ as its Cartan involution [2, Sec. 5]. We also have the existence of a real form $\mathfrak{g}$ of $\stackrel{\circ}{L}$, with $\stackrel{\circ}{\theta}$ as its Cartan

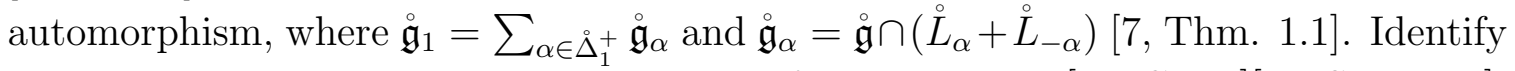
$L$ with its realization as a central extension of a loop algebra [16, Ch. 9][21, Sec. 18.2]. Let us define

$$
\mathfrak{g}=\mathfrak{g}_{\overline{0}} \oplus \sum_{\alpha \in \grave{\Delta}_{1}^{+}} \mathfrak{g}_{\alpha+n \delta}
$$

where

$$
\mathfrak{g}_{\alpha+n \delta}=\left\{t^{n} x_{\alpha}+t^{-n} x_{-\alpha} \mid x_{\alpha}+x_{-\alpha} \in \stackrel{\circ}{\alpha}_{\alpha}\right\}
$$

In our notation, $\mathfrak{g}_{\alpha+n \delta}=\mathfrak{g}_{-\alpha-n \delta}$. By dimension counting on $\mathfrak{g}_{\alpha+n \delta}$, it follows that $\mathfrak{g}$ is a vector space real form of $L$. In order to show that $\mathfrak{g}$ is closed under the bracket, we check that

$$
\left[\mathfrak{g}_{\alpha+n \delta}, \mathfrak{g}_{\beta+m \delta}\right] \subset \mathfrak{g}, \quad \text { for all } \quad \alpha, \beta \in \stackrel{\circ}{\Delta}_{1}^{+}
$$

We divide the computations into two cases.

Case 1: $\beta \neq \pm \alpha$. We have

$$
\begin{aligned}
& {\left[t^{n} x_{\alpha}+t^{-n} x_{-\alpha}, t^{m} x_{\beta}+t^{-m} x_{-\beta}\right]} \\
& =t^{n+m}\left[x_{\alpha}, x_{\beta}\right]+t^{-n+m}\left[x_{-\alpha}, x_{\beta}\right]+t^{n-m}\left[x_{\alpha}, x_{-\beta}\right]+t^{-n-m}\left[x_{-\alpha}, x_{-\beta}\right] \\
& =t^{n+m} x_{\alpha+\beta}+t^{-n-m} x_{-\alpha-\beta}+t^{-n+m} x_{-\alpha+\beta}+t^{n-m} x_{\alpha-\beta} \in \mathfrak{g} .
\end{aligned}
$$

Case 2: $\beta= \pm \alpha$. By [7], there exist elements $z_{ \pm \alpha} \in \stackrel{\circ}{L}_{ \pm \alpha}$ such that $x_{1}=z_{\alpha}+i z_{-\alpha}$, $x_{2}=z_{-\alpha}+i z_{\alpha}$ form a basis for $\stackrel{\mathfrak{g}}{\alpha}_{\alpha}, \alpha \in \stackrel{\triangle}{\Delta}_{1}$ and

$$
\stackrel{\circ}{\theta}\left(x_{1}\right)=-x_{2}, \quad \stackrel{\circ}{\theta}\left(x_{2}\right)=x_{1} .
$$

Hence

$$
X_{1, n}=t^{n} z_{\alpha}+i t^{-n} z_{-\alpha}, \quad X_{2, n}=t^{-n} z_{-\alpha}+i t^{n} z_{\alpha}
$$

form a basis for $\mathfrak{g}_{\alpha+n \delta}$. 
We need to compute $\left[X_{i, n}, X_{j, m}\right]$ for $i, j=1,2$ and $m, n \in \mathbb{Z}_{\geq 0}$. We compute $\left[X_{1, n}, X_{1, m}\right]$, since the other situations are similar. Assume $2 \alpha$ is a root, as otherwise the calculation simplifies further. For $m \neq \pm n$,

$$
\begin{aligned}
{\left[X_{1, n}, X_{1, m}\right] } & =\left[t^{n} z_{\alpha}+i t^{-n} z_{-\alpha}, t^{m} z_{\alpha}+i t^{-m} z_{-\alpha}\right] \\
& =t^{n+m} x_{2 \alpha}-t^{-n-m} x_{-2 \alpha}+i t^{-n+m}\left[z_{-\alpha}, z_{\alpha}\right]+i t^{n-m}\left[z_{\alpha}, z_{-\alpha}\right] \\
& \in \mathfrak{g}_{2 \alpha+(n+m) \delta}+\mathfrak{g}_{\overline{0}},
\end{aligned}
$$

where $x_{ \pm 2 \alpha}=\left[z_{ \pm \alpha}, z_{ \pm \alpha}\right]$. For $m= \pm n$,

$$
\begin{aligned}
{\left[X_{1, n}, X_{1, n}\right] } & =\left[t^{n} z_{\alpha}+i t^{-n} z_{-\alpha}, t^{n} z_{\alpha}+i t^{-n} z_{-\alpha}\right] \\
& =t^{2 n} x_{2 \alpha}-t^{-2 n} x_{-2 \alpha}+i\left[z_{-\alpha}, z_{\alpha}\right]+i c B\left(z_{-\alpha}, z_{\alpha}\right) \\
& +i\left[z_{\alpha}, z_{-\alpha}\right]+i c B\left(z_{\alpha}, z_{-\alpha}\right) \in \mathfrak{g}_{2 \alpha+2 n \delta}+\mathfrak{g}_{\overline{0}} .
\end{aligned}
$$

The brackets $\left[\mathfrak{g}_{\alpha+n \delta}, \mathfrak{g}_{\alpha-n \delta}\right],\left[\mathfrak{g}_{\overline{0}}, \mathfrak{g}_{\alpha+n \delta}\right]$ are carried out in a similar fashion, by using the explicit realization in [2] and in [21, Sec. 18.2]. We have proved (2.2), which implies that $\mathfrak{g}$ is a real form of the affine Lie superalgebra $L$.

It remains to prove that $\theta$ is a Cartan automorphism of $\mathfrak{g}$. To show that $\theta(\mathfrak{g})=\mathfrak{g}$, it is enough to verify that $\theta\left(\mathfrak{g}_{\alpha+n \delta}\right)=\mathfrak{g}_{\alpha+n \delta}$ for all $\alpha \in \stackrel{\circ}{1}_{1}^{+}$. Assume for now $\delta$ compact, that is $\left.\theta\right|_{L_{n \delta}}=1$. By $(2.3)$ we have $\theta\left(z_{\alpha}\right)=-i z_{\alpha}, \theta\left(z_{-\alpha}\right)=i z_{-\alpha}$. Then

$$
\begin{aligned}
& \theta\left(X_{1, n}\right)=\theta\left(t^{n} z_{\alpha}+i t^{-n} z_{-\alpha}\right)=-i t^{n} z_{\alpha}-t^{-n} z_{-\alpha}=-X_{2, n} \in \mathfrak{g}_{\alpha+n \delta}, \\
& \theta\left(X_{2, n}\right)=\theta\left(t^{-n} z_{-\alpha}+i t^{n} z_{\alpha}\right)=i t^{-n} z_{-\alpha}+t^{n} z_{\alpha}=X_{1, n} \in \mathfrak{g}_{\alpha+n \delta} .
\end{aligned}
$$

The computations are similar for $\delta$ non-compact, namely $\left.\theta\right|_{L_{n \delta}}=(-1)^{n} \mathrm{id}$. We have thus shown that $\theta(\mathfrak{g})=\mathfrak{g}$.

Recall that $B$ is an invariant supersymmetric bilinear form on $L[21, \S \S 5.4,18.2]$, [2, §2.3]. In our concrete realization of $L$, we have $B\left(t^{n} X, t^{m} Y\right)=\delta_{n,-m} B(X, Y)$. We consider $B_{\theta}(X, Y)=B(X, \theta(Y))$. Since $\theta_{\overline{0}}$ is a Cartan involution on $\mathfrak{g}_{\overline{0}}$, it follows that $-B_{\theta}$ is positive definite on $\mathfrak{g}_{\overline{0}}$. Hence it is enough to show the same for $-B_{\theta}$ on $\mathfrak{g}_{\alpha+n \delta}$ for $\alpha \in \stackrel{\circ}{\Delta}_{1}$. Using the basis $x_{1}, x_{2} \in \stackrel{\circ}{\mathfrak{g}}_{\alpha}$ and $X_{1, n}, X_{2, n} \in \mathfrak{g}_{\alpha+n \delta}$ of (2.3) and (2.4), a direct calculation shows that

$$
B_{\theta}\left(X_{i, n}, X_{j, n}\right)=B_{\theta}\left(x_{i}, x_{j}\right), \quad i, j=1,2,
$$

keeping in mind $B\left(x_{ \pm \alpha}, x_{ \pm \alpha}\right)=0$ for $x_{ \pm \alpha} \in \stackrel{\mathfrak{g}}{\alpha}_{\alpha}$. So the matrices of $\left.B_{\theta}\right|_{\mathfrak{g}_{\alpha+n \delta}}$ and $\left.B_{\theta}\right|_{\mathfrak{g}_{\alpha}}$ coincide with respect to $X_{1, n}, X_{2, n}$ and $x_{1}, x_{2}$. Since $\left.B_{\theta}\right|_{\mathfrak{g}_{\alpha}}$ is negative definite [7], the same is true for $\left.B_{\theta}\right|_{\mathfrak{g}_{\alpha+n \delta}}$. 
We want to establish the converse of the previous proposition. We start with a lemma.

\section{Lemma 2.3.}

(a) An equal rank real form $\mathfrak{g}$ of $L$ is generated by $\mathfrak{g}$ and $\mathfrak{g}_{\overline{0}}$.

(b) Let $\mathfrak{g}$ and $\mathfrak{g}^{\prime}$ be two equal rank real forms of $L$ such that $\mathfrak{g}=\mathfrak{g}^{\prime}$ and $\mathfrak{g}_{\overline{0}}=\mathfrak{g}_{\overline{0}}^{\prime}$. Then $\mathfrak{g}=\mathfrak{g}^{\prime}$.

Proof. Let $X \in \mathfrak{g}$. Since $L$ is generated by $\stackrel{\circ}{L}$ and $L_{\overline{0}}$, by regarding $X$ as an element of $L$, we can write $X=\sum_{j}\left[X_{j}, Y_{j}\right]$, where $X_{j}, Y_{j} \in \stackrel{\circ}{L} \cup L_{\overline{0}}$. Since $\mathfrak{g}$ and $\mathfrak{g}_{\overline{0}}$ are real forms of $\stackrel{\circ}{L}$ and $L_{\overline{0}}$ respectively, we have $X_{j}=p_{j}+i q_{j}$ and $Y_{j}=r_{j}+i s_{j}$, where $p_{j}, q_{j}, r_{j}, s_{j} \in \mathfrak{g} \cup \mathfrak{g}_{\overline{0}}$. Hence $X$ can be expressed as the brackets of $p_{j}, q_{j}, r_{j}, s_{j}$, which implies that $\mathfrak{g}$ is generated by $\mathfrak{g}$ and $\mathfrak{g}_{\overline{0}}$. This proves part (a). Part (b) is an immediate consequence of part (a).

The next proposition uses the fact that an inner automorphism on $L_{\overline{0}}$ extends to an automorphism on $L$. The proof of this fact has a different flavor, so we discuss it separately as Theorem 6.1 in Section 6 .

Proposition 2.4. Let $\mathfrak{g}$ be an equal rank real form of $L$. Then there exists $\theta \in$ $\operatorname{aut}_{2,4}(L)$ of equal rank, which is a Cartan automorphism of $\mathfrak{g}$.

Proof. Let $\mathfrak{g}$ be an equal rank real form of $L$. We apply the arguments of [2, p. 227228]. There exists an inner automorphism $\tau_{\overline{0}}$ of $L_{\overline{0}}$ which maps a Cartan subalgebra of $\mathfrak{g}_{\overline{0}}$ to $\operatorname{span}_{\mathbb{R}}\left\{i H_{\alpha}, \alpha \in \Delta_{\overline{0}}\right\}$. Let $\mathfrak{g}_{\overline{0}}^{\prime \prime}=\tau_{\overline{0}}\left(\mathfrak{g}_{\overline{0}}\right)$. Then there exist root vectors $x_{ \pm \alpha} \in L_{ \pm \alpha}$ such that

$$
\mathfrak{g}_{0}^{\prime \prime}=\operatorname{span}_{\mathbb{R}}\left\{i H_{\alpha}, t^{n} x_{\alpha}+t^{-n} x_{-\alpha}, \alpha \in \Delta_{\overline{0}}\right\} .
$$

By Theorem 6.1, we can extend $\tau_{\overline{0}}$ to an inner automorphism $\tau$ of $L$. Hence we may assume that $\mathfrak{g}_{\overline{0}}=\mathfrak{g}_{\overline{0}}^{\prime \prime}$. Define

$$
\mathfrak{g}^{\prime}=\mathfrak{g}_{\overline{0}}+\sum_{\alpha \in \grave{\Delta}_{1}} \mathfrak{g}_{\alpha+n \delta}^{\prime}
$$

where $\mathfrak{g}_{\alpha+n \delta}^{\prime}$ are defined as in (2.1). By similar argument as in Proposition 2.2, it follows that $\mathfrak{g}^{\prime}$ is a real form of $L$.

We now define $\theta$ for $\mathfrak{g}^{\prime}$. The Cartan involution $\theta_{\overline{0}}$ of $\mathfrak{g}_{0}^{\prime}$ has been given by [2], and we want to extend it to $\mathfrak{g}^{\prime}$. We define $\theta$ on $\mathfrak{g}_{\alpha+n \delta}^{\prime}$ by the basis elements $X_{1, n}, X_{2, n}$ of (2.4). Namely, if $\delta$ is compact,

$$
\theta\left(X_{1, n}\right)=-X_{2, n}, \quad \theta\left(X_{2, n}\right)=X_{1, n}
$$

While if $\delta$ is non-compact,

$$
\theta\left(X_{1, n}\right)=-(-1)^{n} X_{2, n}, \quad \theta\left(X_{2, n}\right)=(-1)^{n} X_{1, n}
$$


because $\left.\theta\right|_{L_{n \delta}}=(-1)^{n}$ id. This leads to $\theta \in \operatorname{aut}_{2,4}(L)$. By the proof of Proposition 2.2 , it is a Cartan automorphism of $\mathfrak{g}^{\prime}$. We have two real forms $\mathfrak{g}$ and $\mathfrak{g}^{\prime}$, with $\mathfrak{g}_{\overline{0}}=\mathfrak{g}_{\overline{0}}^{\prime}$ and $\mathfrak{g}=\mathfrak{g}^{\prime}$. By Lemma $2.3, \mathfrak{g}=\mathfrak{g}^{\prime}$.

We say that two equal rank real forms are equivalent if they are isomorphic. We say that two equal rank $\theta, \theta^{\prime} \in \operatorname{aut}_{2,4}(L)$ are equivalent if there exists an inner automorphism $\sigma$ on $L$ (see Section 6) such that $\theta^{\prime}=\sigma \theta \sigma^{-1}$. We next show that the correspondence between equal rank real forms and Cartan automorphisms is indeed one-to-one up to equivalence.

Theorem 2.5. Up to equivalence, we have a bijective correspondence between:

(a) Equal rank real forms $\mathfrak{g}$ of $L$,

(b) Equal rank $\theta \in \operatorname{aut}_{2,4}(L)$.

In this correspondence, $\theta$ is a Cartan automorphism of $\mathfrak{g}$.

Proof. If we have an equal rank $\theta \in \operatorname{aut}_{2,4}(L)$, then by Proposition 2.2, there exists an equal rank real form $\mathfrak{g}$ such that $\theta$ is a Cartan automorphism of $\mathfrak{g}$. Suppose that $\theta$ is equivalent to $\theta^{\prime}$, namely $\theta^{\prime}=\sigma \theta \sigma^{-1}$. Let $\mathfrak{g}^{\prime}$ be the real form obtained from $\theta^{\prime}$ via Proposition 2.2. We have $\stackrel{\mathfrak{g}}{\prime}^{\prime}=\sigma\left(\stackrel{\mathfrak{g}}{)}\right.$ ) and $\mathfrak{g}_{\overline{0}}^{\prime}=\sigma\left(\mathfrak{g}_{\overline{0}}\right)$. By Lemma 2.3, $\mathfrak{g}^{\prime}=\sigma(\mathfrak{g})$.

Conversely, let $\mathfrak{g}$ be an equal rank real form of $L$. By Proposition 2.4, it has an equal rank Cartan automorphism $\theta \in \operatorname{aut}_{2,4}(L)$. If we have two isomorphic real forms $\mathfrak{g}$ and $\mathfrak{g}^{\prime}=\sigma(\mathfrak{g})$, with $\theta$ and $\theta^{\prime}$ constructed as in Proposition 2.4, then we can extend $\sigma$ by $\mathbb{C}$-linearity to an automorphism $\sigma$ on $L$. So we have the automorphisms $\theta$ and $\sigma^{-1} \theta^{\prime} \sigma$ which agree on $L_{\overline{0}}$ and $\stackrel{\circ}{L}$. Since $L_{\overline{0}}$ and $\stackrel{\circ}{L}$ generate $L$, we have $\theta=\sigma^{-1} \theta^{\prime} \sigma$. 


\section{Cartan automorphisms and Vogan diagrams}

In this section we establish a bijective correspondence between the two items in Theorem 1.1(b,c), namely the equivalence classes of equal rank $\operatorname{aut}_{2,4}(L)$ and Vogan diagrams. We then complete the proofs of Theorem 1.1 and Corollary 1.2. We introduce several concepts regarding Dynkin and Vogan diagrams, and they will be clarified by some examples in Section 5.

Let $L$ be an affine non-twisted Lie superalgebra. Let $\theta \in \operatorname{aut}_{2,4}(L)$ be of equal rank, namely $\theta=1$ on a Cartan subalgebra of $L$. Let $\Delta=\Delta_{\overline{0}} \cup \Delta_{\overline{1}}$ be the roots. The root spaces $L_{\alpha}$ belong to eigenspaces of $\theta$, and we use the notation

$$
\theta_{\alpha}=\text { eigenvalue of } \theta \text { on } L_{\alpha} \in \begin{cases}\{ \pm 1\} & \text { if } \alpha \in \Delta_{\overline{0}} \\ \{ \pm i\} & \text { if } \alpha \in \Delta_{\overline{1}}\end{cases}
$$

For all $\alpha, \beta \in \Delta$ and $x_{\alpha} \in L_{\alpha}, x_{\beta} \in L_{\beta}$, we have $\left[\theta x_{\alpha}, \theta x_{\beta}\right]=\theta\left[x_{\alpha}, x_{\beta}\right]$. So

$$
\theta_{\alpha} \theta_{\beta}=\theta_{\alpha+\beta}, \theta_{\alpha}^{-1}=\theta_{-\alpha}
$$

if $\alpha+\beta \in \Delta$. We now discuss the diagrammatic method to represent $\theta$.

Let $\mathrm{D}$ be a diagram with positive integers $\left\{a_{\alpha}\right\}_{\mathrm{D}}$ taken from [5, Fig. 1]. Let $\Pi=$ $\stackrel{\circ}{\Pi} \cup\{\delta+\eta\}$ be a simple system of $\stackrel{\circ}{L}, \delta$ generates the imaginary roots $\{n \delta ; 0 \neq n \in \mathbb{Z}\}$, and $\eta$ is the lowest root of $\mathrm{I}^{\circ}$. There are two ways to let the vertices of $\mathrm{D}$ represent roots,

(a) affine viewpoint: $\mathrm{D}$ represents $\prod^{\circ} \cup\{\delta+\eta\} \subset \Delta, \sum_{\mathrm{D}} a_{\alpha} \alpha=\delta$

(b) finite viewpoint: D represents $\prod^{\circ} \cup\{\eta\} \subset \stackrel{\circ}{\Delta}, \sum_{\mathrm{D}} a_{\alpha} \alpha=0$.

We take now the affine viewpoint. Write $\mathrm{D}=\mathrm{D}_{\overline{0}} \cup \mathrm{D}_{\overline{1}}$ for the white and dark (i.e. grey or black) vertices respectively. We add a vertex to each connected component of $\mathrm{D}_{\overline{0}}$ to form its extended Dynkin diagram $\widehat{\mathrm{D}_{\overline{0}}}$. Here $\mathrm{D}_{\overline{0}}$ is the Dynkin diagram of ${\stackrel{\circ}{L_{\overline{0}}}}_{\overline{0}}$, and $\widehat{D_{\overline{0}}}$ is the Dynkin diagram of $L_{\overline{0}}$. Its vertices carry positive integers $\left\{b_{\alpha}\right\}_{\widehat{D_{\overline{0}}}}[16$, Table Aff 1]. Each connected component $K$ of $\widehat{\mathrm{D}_{\overline{0}}}$ represents a simple system of an ideal of $L_{\overline{0}}$, and

$$
\sum_{\mathrm{D}} a_{\alpha} \alpha=\sum_{K} b_{\alpha} \alpha=\delta
$$

by the very definitions of the labels $a_{\alpha}, b_{\alpha}$. We will give examples of $\mathrm{D}$ in Figure 2,

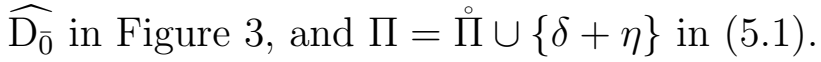

There are various simple systems $\Pi$ of $L$, and we always assume that our $\Pi$ has as few odd roots as possible. When we can choose $\Pi$ with one (resp. two) odd root, we say that $L$ is of type 2 (resp. 1 ). In this way its Dynkin diagram appears as in $[5$, Fig. 1]. 
A Vogan diagram on $\mathrm{D}, \mathrm{D}_{\overline{0}}$ or $\widehat{\mathrm{D}_{\overline{0}}}$ is a function $c$ which assigns \pm 1 to white vertices, and $\pm i$ to dark vertices. We specify $c$ by circling the vertices where $c$ attains the values -1 and $-i$. The general definitions of Vogan diagrams elsewhere involve diagram involutions [17, VI-8][7, Def. 1.2]. But here we do not need diagram involutions because our real forms are of equal rank.

Let $\mathcal{V}(\cdot)$ denote Vogan diagrams. We say that $c \in \mathcal{V}(\mathrm{D})$ represents $\theta \in \operatorname{aut}_{2,4}(L)$ if the vertices of $\mathrm{D}$ represent $\Pi$, and $c_{\alpha}=\theta_{\alpha}$ for all $\alpha \in \mathrm{D}$, namely

(a) $\alpha$ white uncircled $\Longleftrightarrow \theta_{\alpha}=1$,

(b) $\alpha$ white circled $\Longleftrightarrow \theta_{\alpha}=-1$,

(c) $\alpha$ dark uncircled $\Longleftrightarrow \theta_{\alpha}=i$,

(d) $\alpha$ dark circled $\Longleftrightarrow \theta_{\alpha}=-i$.

We similarly say that $c \in \mathcal{V}\left(\mathrm{D}_{\overline{0}}\right)$ (resp. $c \in \mathcal{V}\left(\widehat{\mathrm{D}_{\overline{0}}}\right)$ ) represents $\theta \in \operatorname{inv}\left({\stackrel{\circ}{L_{\overline{0}}}}_{\overline{0}}\right)$ (resp. $\left.\theta \in \operatorname{inv}\left(L_{\overline{0}}\right)\right)$ if $\mathrm{D}_{\overline{0}}$ (resp. $\widehat{\mathrm{D}_{\overline{0}}}$ ) represents a simple system of $\stackrel{\circ}{L}_{\overline{0}}$ (resp. $L_{\overline{0}}$ ) such that (3.5) holds for its vertices. We will give examples of $\mathcal{V}(\mathrm{D})$ in Figure 4 , and $\mathcal{V}\left(\widehat{\mathrm{D}_{\overline{0}}}\right)$ in Figure 5. We define

$$
\chi: \mathcal{V}(\mathrm{D}) \longrightarrow\{ \pm 1\}, \chi(c)=\prod_{\mathrm{D}} c_{\alpha}^{a_{\alpha}}
$$

Since D either has two grey vertices $\beta, \gamma$ where $a_{\beta}=a_{\gamma}=1$ or one dark vertex $\gamma$ where $a_{\gamma}=2$, the value of $\chi(c)$ in $(3.6)$ is indeed \pm 1 . We let $\mathcal{V}(\mathrm{D})_{ \pm}=\chi^{-1}( \pm 1)$ and have the disjoint union

$$
\mathcal{V}(\mathrm{D})=\mathcal{V}(\mathrm{D})_{+} \cup \mathcal{V}(\mathrm{D})_{-}
$$

Let $c$ be a Vogan diagram on $\mathrm{D}, \mathrm{D}_{\overline{0}}$ or $\widehat{\mathrm{D}_{\overline{0}}}$. If $\alpha$ is a white circled vertex, we let $F_{\alpha} c$ be another Vogan diagram given by

$$
\begin{aligned}
F_{\alpha}: & \text { reverse the circlings of all vertices } \beta \text { adjacent to } \alpha, \\
& \text { except when } \beta \text { is a long root joint to } \alpha \text { by a double edge. }
\end{aligned}
$$

We also define $F_{\overline{1}} c$ to be

$$
F_{\overline{1}} \text { : reverse the circlings of all dark vertices. }
$$

If $c$ represents $\theta$ with respect to a simple system $\Pi$, then $F_{\alpha} c$ represents $\theta$ with respect to $r_{\alpha} \Pi$, where $r_{\alpha}: \Delta \longrightarrow \Delta$ is the reflection defined by $\alpha \mapsto-\alpha$. Also, $F_{\overline{1}} c$ represents $\theta$ with respect to $-\Pi$ due to (3.1) and (3.2).

We let $\mathcal{F}$ denote a sequence $\left(F_{1}, \ldots, F_{n}\right)$, where each $F_{i}$ belongs to $(3.7),(3.8)$ or is a diagram automorphism. 
Definition 3.1. Let $c, c^{\prime} \in \mathcal{V}(X)$, where $X \in\left\{\mathrm{D}, \mathrm{D}_{\overline{0}}, \widehat{\mathrm{D}_{\overline{0}}}\right\}$. We say that $c$ and $c^{\prime}$ are equivalent if one of the following holds.

(a) There exists a sequence $\mathcal{F}$ which transforms $c$ to $c^{\prime}$.

(b) $X=\mathrm{D}$ and $\chi(c)=\chi\left(c^{\prime}\right)=-1$.

We will illustrate Definition 3.1(a) by Figure 4(a,b). Definition 3.1(b) may appear strange, as $c, c^{\prime} \in \mathcal{V}(\mathrm{D})_{-}$are automatically equivalent without the need to have $\mathcal{F} c=c^{\prime}$. We shall explain this phenomena in Observation 5.1.

By (3.4) and (3.5), if $c \in \mathcal{V}(\mathrm{D})$ represents $\theta \in \operatorname{aut}_{2,4}(L)$, then $\prod_{\mathrm{D}} c_{\alpha}^{a_{\alpha}}=\theta_{\delta}$. Therefore,

$$
c \in \mathcal{V}(\mathrm{D})_{ \pm} \Longleftrightarrow \theta_{\delta}= \pm 1
$$

For each connected component $K$ of $\widehat{\mathrm{D}_{\overline{0}}}$, we define

$$
\chi_{K}: \mathcal{V}\left(\widehat{\mathrm{D}_{\overline{0}}}\right) \longrightarrow\{ \pm 1\}, \chi_{K}(c)=\prod_{K} c_{\alpha}^{b_{\alpha}} .
$$

We also define

$$
\mathcal{V}\left(\widehat{\mathrm{D}_{\overline{0}}}\right)_{ \pm}=\left\{c \in \mathcal{V}\left(\widehat{\mathrm{D}_{\overline{0}}}\right) ; \chi_{K}(c)= \pm 1 \text { for all } K\right\}
$$

One has $\mathcal{V}\left(\widehat{\mathrm{D}_{\overline{0}}}\right)=\mathcal{V}\left(\widehat{\mathrm{D}_{\overline{0}}}\right)_{+} \cup \mathcal{V}\left(\widehat{\mathrm{D}_{\overline{0}}}\right)_{-}$if and only if $\widehat{\mathrm{D}_{\overline{0}}}$ is connected. By (3.4) and (3.5), if $c \in \mathcal{V}\left(\widehat{\mathrm{D}_{\overline{0}}}\right)$ represents $\theta \in \operatorname{inv}\left(L_{\overline{0}}\right)$, then $\prod_{K} c_{\alpha}^{b_{\alpha}}=\theta_{\delta}$. Therefore,

$$
c \in \mathcal{V}\left(\widehat{\mathrm{D}_{\overline{0}}}\right)_{ \pm} \Longleftrightarrow \theta_{\delta}= \pm 1 \text {. }
$$

Define the natural map

$$
\mathcal{V}(\mathrm{D})_{ \pm} \longrightarrow \mathcal{V}\left(\widehat{\mathrm{D}_{\overline{0}}}\right)_{ \pm}
$$

Namely, given $c \in \mathcal{V}(D)_{ \pm}$, we first omit the dark vertices to get a member of $\mathcal{V}\left(\mathrm{D}_{\overline{0}}\right)$. We then add vertices of $\widehat{\mathrm{D}_{\overline{0}}} \backslash \mathrm{D}_{\overline{0}}$, and their circlings are determined by $\chi_{K}= \pm 1$. By (3.9) and (3.10), if $c \in \mathcal{V}(\mathrm{D})_{ \pm}$represents $\theta \in$ aut $_{2,4}(L)$, then its image under (3.11) represents the restriction of $\theta$ to $L_{\overline{0}}$. We will illustrate (3.11) by examples in (5.2) and (5.3).

The restriction map

$$
\mathcal{V}\left(\widehat{\mathrm{D}_{\overline{0}}}\right)_{+} \longrightarrow \mathcal{V}\left(\mathrm{D}_{\overline{0}}\right)
$$

is defined by omitting the vertices of $\widehat{\mathrm{D}_{\overline{0}}} \backslash \mathrm{D}_{\overline{0}}$.

One checks that diagram automorphisms as well as $F_{\alpha}$ and $F_{\overline{1}}$ of (3.7) and (3.8) preserve $\chi$ and $\chi_{K}$. Hence $\mathcal{V}(\mathrm{D})_{ \pm}$and $\mathcal{V}\left(\widehat{\mathrm{D}_{\overline{0}}}\right)_{ \pm}$are unions of equivalence classes. The next proposition relates these equivalence classes with the maps (3.11) and (3.12). 


\section{Proposition 3.2.}

(a) The restriction map $\mathcal{V}\left(\widehat{\mathrm{D}_{\overline{0}}}\right)_{+} \longrightarrow \mathcal{V}\left(\mathrm{D}_{\overline{0}}\right)$ leads to a bijection between their equivalence classes.

(b) $\mathcal{V}\left(\widehat{\mathrm{D}_{\overline{0}}}\right)_{-}$is one equivalence class.

(c) The natural map $\mathcal{V}(\mathrm{D})_{ \pm} \longrightarrow \mathcal{V}\left(\widehat{\mathrm{D}_{\overline{0}}}\right)_{ \pm}$leads to a bijection between their equivalence classes.

Proof. Two members of $\mathcal{V}\left(\mathrm{D}_{\overline{0}}\right)$ or $\mathcal{V}\left(\widehat{\mathrm{D}_{\overline{0}}}\right)$ are equivalent if and only if their restrictions to each connected component are equivalent. So it suffices to study a connected component of $\mathrm{D}_{\overline{0}}$, and ignore the Vogan diagrams with no circled vertex because they are not equivalent to any other diagram.

Parts (a) and (b) are verified directly by comparing [11, Table 1] and [12, Table 1]. For example, let $L_{\overline{0}}=F_{4}$. Then $\mathcal{V}\left(\mathrm{D}_{\overline{0}}\right)$ has two equivalence classes [11, Table 1], say $S_{1}$ and $S_{2}$. Also, $\mathcal{V}\left(\widehat{\mathrm{D}_{\overline{0}}}\right)$ has three equivalence classes [12, Table 1], where one of them is $\mathcal{V}\left(\widehat{\mathrm{D}_{\overline{0}}}\right)_{-}$, and the other two correspond to $S_{1}$ and $S_{2}$ by $\mathcal{V}\left(\widehat{\mathrm{D}_{\overline{0}}}\right)_{+} \longrightarrow \mathcal{V}\left(\mathrm{D}_{\overline{0}}\right)$. Similarly if $L_{\overline{0}}=G_{2}$, then $\mathcal{V}\left(\mathrm{D}_{\overline{0}}\right)$ has one equivalence class [11, Table 1$]$, and $\mathcal{V}\left(\widehat{\mathrm{D}_{\overline{0}}}\right)$ has two equivalence classes $\mathcal{V}\left(\widehat{\mathrm{D}_{\overline{0}}}\right)_{+}$and $\mathcal{V}\left(\widehat{\mathrm{D}_{\overline{0}}}\right)_{-}[12$, Table 1$]$.

Finally we prove part $(\mathrm{c})$. The case of $\mathcal{V}(\mathrm{D})_{-} \longrightarrow \mathcal{V}\left(\widehat{\mathrm{D}_{\overline{0}}}\right)_{-}$is trivial, because by Definition 3.1(b) and part (b) of this proposition, each of $\mathcal{V}(\mathrm{D})_{-}$and $\mathcal{V}\left(\widehat{\mathrm{D}_{\overline{0}}}\right)_{-}$is a single equivalence class. So we consider only $\mathcal{V}(\mathrm{D})_{+} \longrightarrow \mathcal{V}\left(\widehat{\mathrm{D}_{\overline{0}}}\right)_{+}$.

Let $c_{1}, c_{2} \in \mathcal{V}(\mathrm{D})_{+}$, and let $c_{1}^{\prime}, c_{2}^{\prime} \in \mathcal{V}\left(\widehat{\mathrm{D}_{\overline{0}}}\right)_{+}$be their images under $\mathcal{V}(\mathrm{D})_{+} \longrightarrow$ $\mathcal{V}\left(\widehat{\mathrm{D}_{\overline{0}}}\right)_{+}$. Suppose that $c_{1} \sim c_{2}$. By Definition 3.1(a), there exists a sequence $\mathcal{F}$ such that $\mathcal{F} c_{1}=c_{2}$. Then $\mathcal{F} c_{1}^{\prime}, c_{2}^{\prime} \in \mathcal{V}\left(\widehat{\mathrm{D}_{\overline{0}}}\right)+$ agree on $\mathrm{D}_{\overline{0}}$.

Let $K$ be a connected component of $\widehat{\mathrm{D}_{\overline{0}}}$. Write $\{\beta\}=K \backslash\left(K \cap \mathrm{D}_{\overline{0}}\right)$, where $b_{\beta}=1$. We have

$$
1=\chi_{K}\left(\mathcal{F} c_{1}^{\prime}\right)=\prod_{K}\left(\mathcal{F} c_{1}^{\prime}\right)_{\alpha}^{b_{\alpha}}=\left(\mathcal{F} c_{1}^{\prime}\right)_{\beta} \prod_{K \cap D_{\overline{0}}}\left(\mathcal{F} c_{1}^{\prime}\right)_{\alpha}^{b_{\alpha}} .
$$

We repeat (3.13) on $c_{2}^{\prime}$. Since $\mathcal{F} c_{1}^{\prime}$ and $c_{2}^{\prime}$ agree on $\mathrm{D}_{\overline{0}}$, by $(3.13),\left(\mathcal{F} c_{1}^{\prime}\right)_{\beta}=\left(c_{2}^{\prime}\right)_{\beta}$. Hence $\mathcal{F} c_{1}^{\prime}=c_{2}^{\prime}$, namely $c_{1}^{\prime} \sim c_{2}^{\prime}$

Conversely, suppose that $c_{1}^{\prime} \sim c_{2}^{\prime}$. By part (a), their restrictions to $\mathrm{D}_{\overline{0}}$ are also equivalent. So there exists a sequence $\mathcal{F}$, consisting of diagram automorphism on $\mathrm{D}_{\overline{0}}$ and $F_{\alpha}$ where $\alpha \in \mathrm{D}_{\overline{0}}$ (without $\alpha \in \widehat{\mathrm{D}_{\overline{0}}} \backslash \mathrm{D}_{\overline{0}}$ ), such that $\mathcal{F} c_{1}^{\prime}$ and $c_{2}^{\prime}$ agree on $\mathrm{D}_{\overline{0}}$. Hence $\mathcal{F} c_{1}$ and $c_{2}$ agree on $\mathrm{D}_{\overline{0}}$. This property, together with $\chi\left(\mathcal{F} c_{1}\right)=\chi\left(c_{2}\right)=1$, imply that

$$
\prod_{\mathrm{D}_{\overline{1}}}\left(\mathcal{F} c_{1}\right)_{\alpha}^{a_{\alpha}}=\prod_{\mathrm{D}_{\overline{1}}}\left(c_{2}\right)_{\alpha}^{a_{\alpha}}
$$

We have either $\mathrm{D}_{\overline{1}}=\{\gamma\}$ with $a_{\gamma}=2$, or $\mathrm{D}_{\overline{1}}=\{\beta, \gamma\}$ with $a_{\beta}=a_{\gamma}=1$. So (3.14) implies that $\mathcal{F} c_{1}$ and $c_{2}$ are either the same or opposite on $\mathrm{D}_{\overline{1}}$. In the former case, $\mathcal{F} c_{1}=c_{2}$. In the latter case, $F_{\overline{1}} \mathcal{F} c_{1}=c_{2}$ by (3.8). Hence $c_{1} \sim c_{2}$. 
Theorem 3.3. Let $c, c^{\prime} \in \mathcal{V}(\mathrm{D})$ represent $\theta, \theta^{\prime} \in \operatorname{aut}_{2,4}(L)$ respectively. Then $c \sim c^{\prime}$ if and only if $\theta \sim \theta^{\prime}$.

Proof. Given $c \in \mathcal{V}(\mathrm{D})$, we let $\widehat{c}_{\overline{0}} \in \mathcal{V}\left(\widehat{\mathrm{D}_{\overline{0}}}\right)$ denote its image under (3.11). We also let $c_{\overline{0}} \in \mathcal{V}\left(\mathrm{D}_{\overline{0}}\right)$ be its restriction to $\mathrm{D}_{\overline{0}}$.

Let $\theta, \theta^{\prime} \in \operatorname{aut}_{2,4}(L)$ be represented by $c, c^{\prime} \in \mathcal{V}(\mathrm{D})$ respectively. Suppose that $\theta \sim \theta^{\prime}$. We have $\theta_{\overline{0}} \sim \theta_{\overline{0}}^{\prime}$, hence $\widehat{c}_{\overline{0}} \sim \widehat{c}_{\overline{0}}^{\prime}\left[2\right.$, Sec. 5]. By Proposition 3.2(c), $c \sim c^{\prime}$.

Conversely, suppose that $c \sim c^{\prime}$. By Proposition 3.2(c), $\widehat{c}_{\overline{0}} \sim \widehat{c}_{\overline{0}}^{\prime}$. It implies that $\theta_{\overline{0}} \sim \theta_{\overline{0}}^{\prime}$, namely there exists an inner automorphism $\sigma_{\overline{0}}$ on $L_{\overline{0}}$ such that $\sigma_{\overline{0}} \theta_{\overline{0}} \sigma_{\overline{0}}^{-1}=\theta_{\overline{0}}^{\prime}$ [2, Sec. 5]. By Theorem 6.1, $\sigma_{\overline{0}}$ extends to an $L$-automorphism $\sigma$, so

$$
\left(\sigma \theta \sigma^{-1}\right)_{\overline{0}}=\theta_{\overline{0}}^{\prime}
$$

Condition (3.15) and $\chi(c)=\chi\left(c^{\prime}\right)$ imply that $\prod_{\mathrm{D}_{\overline{1}}}\left(\sigma \theta \sigma^{-1}\right)_{\alpha}^{a_{\alpha}}=\prod_{\mathrm{D}_{\overline{1}}}\left(\theta^{\prime}\right)_{\alpha}^{a_{\alpha}}$. Similar to the arguments of (3.14), it implies that one of the following holds:

(a) $\left(\sigma \theta \sigma^{-1}\right)_{\alpha}=\theta_{\alpha}^{\prime}$ for all $\alpha \in \Pi_{\overline{1}}$,

(b) $\left(\sigma \theta \sigma^{-1}\right)_{\alpha}=-\theta_{\alpha}^{\prime}$ for all $\alpha \in \Pi_{\overline{1}}$.

If (3.16)(a) holds, then $\sigma \theta \sigma^{-1}=\theta^{\prime}$ and we are done. Suppose that (3.16)(b) holds. Then by (3.2), (3.15) and (3.16)(b),

$$
\left(\sigma \theta \sigma^{-1}\right)_{\alpha}=\left(\theta^{\prime}\right)_{-\alpha} \text { for all } \alpha \in \Pi \text {. }
$$

Let $\tau: L \longrightarrow L$ be an isomorphism which stabilizes the Cartan subalgebra and maps each $L_{\alpha}$ to $L_{-\alpha}$. By (3.17),

$$
\left(\tau \sigma \theta \sigma^{-1} \tau^{-1}\right)_{\alpha}=\left(\theta^{\prime}\right)_{\alpha} \text { for all } \alpha \in \Pi .
$$

Hence $\tau \sigma \theta \sigma^{-1} \tau^{-1}=\theta^{\prime}$, namely $\theta \sim \theta^{\prime}$.

Proof of Theorem 1.1.

The correspondence between items (a) and (b) of Theorem 1.1 follows from Theorem 2.5. The correspondence between items (b) and (c) of Theorem 1.1 follows from Theorem 3.3.

Let $\stackrel{\circ}{\Pi}$ be a simple system of $\stackrel{\circ}{L}$, and let $\eta$ be its lowest root. Recall from (3.3) that $\mathrm{D}$ may represent roots by the affine or finite viewpoint. We have been adopting the affine viewpoint, so that every member of $\mathcal{V}(\mathrm{D})$ represents aut $_{2,4}(L)$ in Theorem 1.1. If we adopt the finite viewpoint, then $\mathrm{D}=\stackrel{\circ}{\Pi} \cup\{\eta\}$ are linearly dependent. In this case condition (3.2) says that a member of $\mathcal{V}(\mathrm{D})$ needs to satisfy an additional condition in order to represent $\operatorname{aut}_{2,4}(\stackrel{\circ}{L})$. This condition is $\chi=1$, as stated by the following theorem. 
Theorem 3.4. Up to equivalence, there is a bijective correspondence among:

(a) Equal rank real forms $\mathfrak{s}$ of $\stackrel{\circ}{L}$,

(b) Equal rank $\stackrel{\circ}{\dot{\theta}} \in \operatorname{aut}_{2,4}(\stackrel{\circ}{L})$,

(c) Vogan diagrams $c \in \mathcal{V}(\mathrm{D})_{+}$.

In this correspondence, D represents $\Pi^{\circ} \cup\{\eta\}$. Also, $\stackrel{\circ}{\theta}$ is a Cartan automorphism of $\mathfrak{s}$, and c represents $\stackrel{\circ}{\theta}$.

Proof. The proof is given by [7, Thms. 1.1,1.3]. This theorem is also valid for nonequal rank $\mathfrak{s}$ and $\stackrel{\circ}{\theta}$, in which case diagram involutions with extra conditions are added to $\mathcal{V}(\mathrm{D})$.

Let $\operatorname{aut}_{2,4}(L)_{+}$and $\operatorname{aut}_{2,4}(L)_{-}$denote the equal rank automorphisms $\theta \in \operatorname{aut}_{2,4}(L)$ with $\theta_{\delta}=1$ and $\theta_{\delta}=-1$ respectively.

Proof of Corollary 1.2.

Let $\mathfrak{s}$ be an equal rank real form of $\stackrel{\circ}{L}$. By Theorem 3.4, it has an equal rank Cartan automorphism $\stackrel{\circ}{\theta} \in \operatorname{aut}_{2,4}(\stackrel{\circ}{L})$. If $\stackrel{\circ}{\theta}$ extends to an equal rank $\theta \in \operatorname{aut}_{2,4}(L)$, then $\theta=1$ on a Cartan subalgebra of $L$, and hence $\theta_{\delta}= \pm 1$. Conversely, the condition $\theta_{\delta}= \pm 1$ uniquely determines an extension $\theta \in \operatorname{aut}_{2,4}(L)_{ \pm}$by applying $(3.2)$ to the eigenvalues $\left\{\theta_{\alpha} ; \alpha \in \stackrel{\circ}{\Pi} \cup\{\delta\}\right\}$. We conclude that $\stackrel{\circ}{\theta}$ has exactly one extension to each of $\operatorname{aut}_{2,4}(L)_{ \pm}$. By Theorem 1.1, they are Cartan automorphisms of equal rank real forms $\widehat{\mathfrak{s}}_{ \pm}$of $L$. This proves the first statement of the corollary.

Conversely, let $\mathfrak{g}$ be an equal rank real form of $L$. By Theorem 1.1, it has a Cartan automorphism $\theta \in \operatorname{aut}_{2,4}(L)_{ \pm}$. By Theorem 3.4, the restriction of $\theta$ to $\stackrel{\circ}{L}$ is a Cartan automorphism of an equal rank real form $\mathfrak{s}$ of $\stackrel{\circ}{L}$. We have $\mathfrak{g}=\widehat{\mathfrak{s}}_{ \pm}$. This proves the second statement of the corollary.

By Theorems 1.1 and 3.4, the equivalence classes of $\operatorname{aut}_{2,4}(L)_{+}$and equal rank $\operatorname{aut}_{2,4}(\stackrel{\circ}{L})$ both correspond to equivalence classes of $\mathcal{V}(\mathrm{D})_{+}$. This proves $(1.2)(\mathrm{a})$.

The equivalence classes of $\operatorname{aut}_{2,4}(L)_{-}$correspond to the equivalence classes of $\mathcal{V}(\mathrm{D})_{-}$. The latter has only one equivalence class, by Definition 3.1(c). This proves $(1.2)(\mathrm{b})$.

In the proof of Corollary 1.2, the decomposition of D into $\mathrm{D}^{\circ} \cup\{\varphi\}$ is not canonical, see (5.1) for an example. However, the arguments are independent of the choice of decomposition. 


\section{Hermitian real forms and Vogan diagrams}

In this section, we prove Theorems 1.4 and 1.5, which classify the Hermitian real forms. Recall that admissible positive systems are defined in (1.1). Also, Definition 1.3 says that $\mathfrak{g}$ is Hermitian if $\mathfrak{g} \cap \stackrel{\circ}{L}$ is Hermitian and $\delta$ is compact.

Proof of Theorem 1.4.

Suppose that $\mathfrak{g}$ is Hermitian. Let $\stackrel{\circ}{ }^{+}$be an admissible positive system for $\stackrel{\circ}{L}$, and let $\mathfrak{g}=\mathfrak{k} \oplus \mathfrak{p}$ be a Cartan decomposition $[7,8]$. Define the positive system

$$
\Delta^{+}=\left\{\alpha+n \delta \in \Delta ; \alpha \in \stackrel{\circ}{\Delta}^{+}\right\} \cup\left\{n \delta \in \Delta ; n \in \mathbb{Z}^{+}\right\}
$$

Since $\delta$ is compact, we have

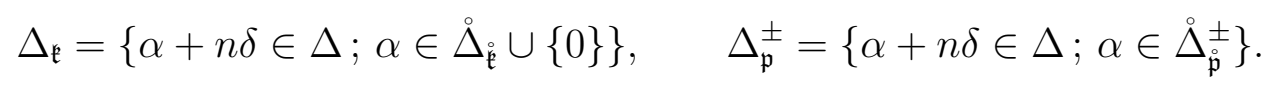

Since $\stackrel{\circ}{ }^{+}$is admissible, these conditions imply that

$$
\Delta_{\mathfrak{k}}+\Delta_{\mathfrak{p}}^{ \pm} \subset \Delta_{\mathfrak{p}}^{ \pm}, \quad \Delta_{\mathfrak{p}}^{ \pm}+\Delta_{\mathfrak{p}}^{ \pm} \notin \Delta
$$

They translate to (1.1), so $\Delta^{+}$is an admissible positive system for $\Delta$.

Conversely, suppose that $\Delta^{+}$is an admissible positive system for $\Delta$. Let $\stackrel{\circ}{ }^{+}=$ $\Delta^{+} \cap \stackrel{\circ}{\Delta}$ and $\Delta_{\overline{0}}^{+}=\Delta^{+} \cap \Delta_{\overline{0}}$. Then $\stackrel{\circ}{ }^{+}$and $\Delta_{\overline{0}}^{+}$are admissible positive systems for $\stackrel{\circ}{\Delta}$ and $\Delta_{\overline{0}}$ respectively. The former implies that $\mathfrak{g}$ is Hermitian [8, Thm. 1.1], and the latter implies that $\delta$ is compact $[9$, Thm. 1.2]. This completes the proof of Theorem 1.4 .

Proof of Theorem 1.5.

By Corollary 1.2, an equal rank real form of $L$ can be expressed as $\mathfrak{g}=\widehat{\mathfrak{s}}_{ \pm}$, where $\mathfrak{s}$ is a real form $\stackrel{\circ}{L}$. By Theorem 1.1, $\mathfrak{g}$ corresponds to $c \in \mathcal{V}(\mathrm{D})$. By $(3.9), \chi(c)=1$ if and only if $\delta$ is compact. So $\mathfrak{g}$ is Hermitian if and only if

(a) $\chi(c)=1$

(b) $\mathfrak{s}$ is Hermitian.

We continue our arguments in three cases.

Case (a): $\stackrel{\circ}{L}$ is of type $A$. All equal rank $\mathfrak{s}$ are Hermitian, so we only need to consider (4.1)(a). Each $\alpha \in \mathrm{D}$ satisfies $a_{\alpha}=1$. Also, $\theta$ has eigenvalues $\pm i$ on the root spaces of the two grey vertices. Therefore, (4.1)(a) is equivalent to having odd number of circled vertices in $\mathrm{D}$. At most one vertex of each connected component of $\mathrm{D}_{\overline{0}}$ needs to be circled [17, Thm. 6.96]. Also, at most one grey vertex needs to be circled due to $F_{\overline{1}}$ of (3.8). This leads to the first row of Figure 1. 
Case (b): $\stackrel{\circ}{L}=C(n)$. Condition (4.1)(b) means that $\stackrel{\circ}{\overline{0}}_{\overline{0}}$ is $\mathfrak{s p}(n-1)$ or $\mathfrak{s p}(n-1, \mathbb{R})$, and they are represented by $\mathcal{V}\left(\mathrm{D}_{\overline{0}}\right)$ in the second row of Figure 1. Similar to Case (a), at most one grey vertex needs to be circled. The circlings on the grey vertices are then forced by $(4.1)(\mathrm{a})$.

Case (c): Remaining cases. Here D has a unique dark vertex $\gamma$, and $a_{\gamma}=2$. By $F_{\overline{1}}$ of (3.8), we may assume that $\gamma$ is not circled. We have $c_{\gamma}^{a_{\gamma}}=i^{2}=-1$, so (4.1)(a) becomes $\prod_{\mathrm{D}_{\overline{0}}} c_{\alpha}^{a_{\alpha}}=-1$. This condition, together with (4.1)(b), are precisely the conditions [7, Def. 1.2(c)] and [8, Def. 3.3], and they lead to all the diagrams in [8, Figs. 2, 4, 5]. We reproduce [8, Figs. 2, 4, 5] in the rest of Figure 1.

Finally we discuss the Iwasawa decomposition on $L$ and the resulting complex structure on $\mathfrak{g} / \mathfrak{k}$. We omit the proof of the next theorem, which is the same as the proofs for [8, Thm. 1.4] and [9, Thm. 1.5]. Let $\mathfrak{g}$ be a Hermitian real form of $L$, and $\mathfrak{h}$ a compact Cartan subalgebra of $\mathfrak{g}$. Let $\mathfrak{h}_{\mathbb{C}}=\mathfrak{h}+i \mathfrak{h}$, and let $\Delta^{+} \subset\left(\mathfrak{h}_{\mathbb{C}}\right)^{*}$ be an admissible positive system. Then $\mathfrak{n}_{\mathbb{C}}=\sum_{\Delta^{+}} L_{\alpha}$ is a nilpotent subalgebra, and $\mathfrak{h}_{\mathbb{C}}+\mathfrak{n}_{\mathbb{C}}$ is a Borel subalgebra.

Theorem 4.1. For a Hermitian real form $\mathfrak{g}$ with compact Cartan subalgebra $\mathfrak{h}$, we have the Iwasawa decomposition $L=\mathfrak{g}+i \mathfrak{h}+\mathfrak{n}_{\mathbb{C}}$. There exists a parabolic subalgebra $\mathfrak{h}_{\mathbb{C}}+\mathfrak{n}_{\mathbb{C}} \subset \mathfrak{p}_{\mathbb{C}} \subset L$ such that the natural map $\mathfrak{g} \hookrightarrow L \longrightarrow L / \mathfrak{p}_{\mathbb{C}}$ leads to an isomorphism $\mathfrak{g} / \mathfrak{k} \cong L / \mathfrak{p}_{\mathbb{C}}$, thereby providing $\mathfrak{g} / \mathfrak{k}$ with a complex structure. 


\section{$5 \quad$ Examples}

In this section, we provide some examples to illustrate several concepts that are discussed in the previous sections.

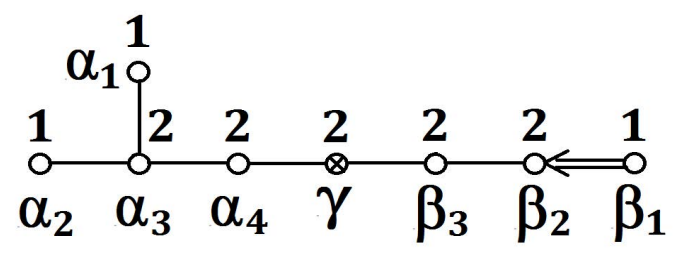

Figure 2: D and $\left\{a_{\alpha}\right\}_{\mathrm{D}}$.

Let $\stackrel{\circ}{L}=D(4,3)$. Figure 2 shows the Dynkin diagram $\mathrm{D}$ of $L$, together with the integers $\left\{a_{\alpha}\right\}_{\mathrm{D}}$ which satisfy (3.4). The vertices represent a simple system $\Pi=$ $\stackrel{\circ}{\Pi} \cup\{\delta+\eta\}$ of $L$, where $\stackrel{\circ}{\Pi}$ is a simple system of $\stackrel{\circ}{L}$ with lowest root $\eta$. Any vertex $\alpha$ with $a_{\alpha}=1$ can represent $\delta+\eta$, so we have two choices here:

(a) $\alpha_{1}=\delta+\eta, \mathrm{D} \backslash\left\{\alpha_{1}\right\}=\stackrel{\circ}{\Pi}$,

(b) $\beta_{1}=\delta+\eta, \mathrm{D} \backslash\left\{\beta_{1}\right\}=\stackrel{\circ}{\Pi}$.

The case of $\alpha_{2}=\delta+\eta$ is similar to (5.1)(a) by diagram symmetry. In any case, our discussions are independent of the choice in (5.1).

Here $\stackrel{\circ}{L}$ is of type 2 , so there is only one grey vertex $\gamma$ in D. The remaining vertices $\mathrm{D}_{\overline{0}}=\left\{\alpha_{i}, \beta_{j}\right\}$ form the Dynkin diagram of $\stackrel{\circ}{L}_{\overline{0}}=D_{4}+C_{3}$. For each connected component of $\mathrm{D}_{\overline{0}}$, we can add a vertex to form the extended Dynkin diagram $[16$, Table Aff1]. The resulting diagram $\widehat{\mathrm{D}_{\overline{0}}}$ is the Dynkin diagram of $L_{\overline{0}}$.
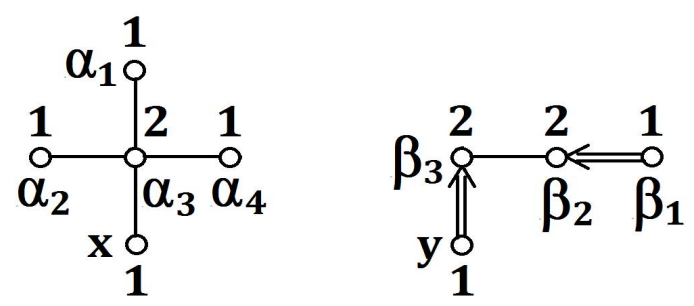

Figure 3: $\widehat{\mathrm{D}_{\overline{0}}}$ and $\left\{b_{\alpha}\right\}_{\widehat{\mathrm{D}_{\overline{0}}}}$.

Figure 3 shows $\widehat{\mathrm{D}_{\overline{0}}}$ together with the integers $b_{\alpha}$ which satisfy (3.4). The added vertices $x$ and $y$ represent the roots of $L_{\overline{0}}$ by

$$
x+\sum_{1}^{4} b_{\alpha_{i}} \alpha_{i}=\delta, y+\sum_{1}^{3} b_{\beta_{i}} \beta_{i}=\delta .
$$




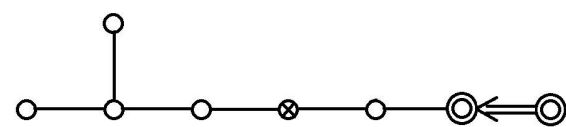

(a)

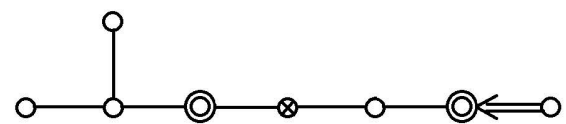

(c)

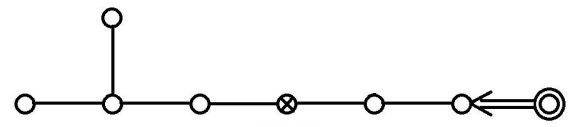

(b)

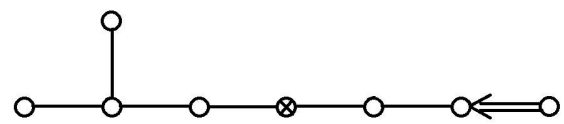

(d)

Figure 4: Equivalence of Vogan diagrams.

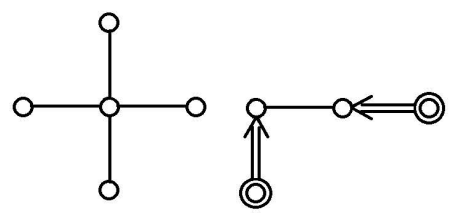

(a)

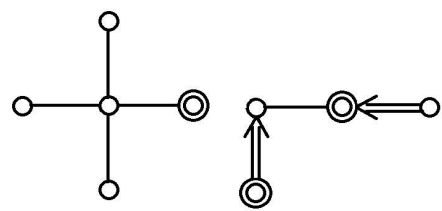

(b)

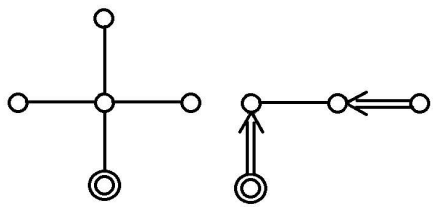

(c)

Figure 5: $\mathcal{V}\left(\widehat{D_{\overline{0}}}\right)_{+}$and $\mathcal{V}\left(\widehat{\mathrm{D}_{\overline{0}}}\right)_{-}$.

We consider the four Vogan diagrams in Figure 4. We denote them by their circled vertices, so for example Figure 4(a) is $c=\left(\beta_{1}, \beta_{2}\right)$. By (3.5) and (3.6), Figure 4(a) satisfies $\chi(c)=c_{\beta_{1}}^{a_{\beta_{1}}} c_{\beta_{2}}^{a_{\beta_{2}}} c_{\gamma}^{a_{\gamma}}=(-1)^{1}(-1)^{2} i^{2}=1$, so $c \in \mathcal{V}(\mathrm{D})_{+}$. We apply $F_{\beta_{1}}$ of (3.7), which reverses the circling of $\beta_{2}$. Namely $F_{\beta_{1}}\left(\beta_{1}, \beta_{2}\right)=\left(\beta_{1}\right)$, which is Figure 4(b). Hence Figure 4(a,b) are equivalent. By Corollary 1.2, they represent a real form $\widehat{\mathfrak{s}}_{+}$of $L$, where $\mathfrak{s}$ is a real form $\stackrel{\circ}{L}$. In this case $\mathfrak{s}_{\overline{0}}=\mathfrak{s o}(8)+\mathfrak{s p}(3, \mathbb{R})$. The map $\mathcal{V}(\mathrm{D})_{+} \longrightarrow \mathcal{V}\left(\widehat{\mathrm{D}_{\overline{0}}}\right)_{+}$of $(3.11)$ leads to

Figure 4(b) $\mapsto$ Figure 5(a).

Figure 4(b) satisfies $\chi=1$. So the circlings of $x$ and $y$ in Figure 5(a) are determined by the conditions $\chi_{K}=1$ for $K=\widehat{D_{4}}$ and $K=\widehat{C_{3}}$.

Before we proceed to study Figure 4(c,d), let us discuss Definition 3.1(b) in the following observation.

Observation 5.1. Hidden diagram symmetry and pushing the hidden button.

Let $c, c^{\prime} \in \mathcal{V}(\mathrm{D})_{-}$, and let $\widehat{c}_{\overline{0}}, \widehat{c}_{\overline{0}}^{\prime}$ be their images under $\mathcal{V}(\mathrm{D})_{-} \longrightarrow \mathcal{V}\left(\widehat{\mathrm{D}_{\overline{0}}}\right)_{-}$of (3.11). By Proposition 3.2(b), $\widehat{c}_{\overline{0}} \sim \widehat{c}_{\overline{0}}^{\prime}$. This motivates Definition 3.1(b), which says that $c \sim c^{\prime}$, so that $\mathcal{V}(\mathrm{D})_{-} \longrightarrow \mathcal{V}\left(\widehat{\mathrm{D}_{\overline{0}}}\right)_{-}$preserves the equivalence relations as stated in Proposition 3.2(c). The condition $\widehat{c}_{\overline{0}} \sim \widehat{c}_{\overline{0}}^{\prime}$ means that $\mathcal{F} \widehat{c}_{\overline{0}}=\widehat{c}_{\overline{0}}^{\prime}$ for some $\mathcal{F}=\left(F_{1}, \ldots, F_{n}\right)$, where each $F_{i}$ belongs to (3.7) or is a diagram automorphism. We may write $\mathcal{F} c=c^{\prime}$, but then some $F_{i}$ may appear as a "hidden diagram symmetry" or pushing a "hidden button" outside D. We shall illustrate them by Figure 4(c,d). 
There is no circled vertex or nontrivial diagram symmetry in Figure 4(d). So Figure $4(\mathrm{c}, \mathrm{d})$ are not related by a sequence of $F_{\alpha}$ for $\alpha \in \mathrm{D}$ or diagram automorphism. But they both satisfy $\chi=-1$, so they are equivalent by Definition 3.1(b). The map $\mathcal{V}(\mathrm{D})_{-} \longrightarrow \mathcal{V}\left(\widehat{\mathrm{D}_{\overline{0}}}\right)_{-}$of $(3.11)$ satisfies

$$
\text { Figure 4(c) } \mapsto \text { Figure 5(b), Figure 4(d) } \mapsto \text { Figure 5(c). }
$$

The circlings of vertices $x$ and $y$ in Figure $5(\mathrm{~b}, \mathrm{c})$ are determined by the conditions $\chi_{K}=-1$ for $K=\widehat{D_{4}}$ and $K=\widehat{C_{3}}$. The $\widehat{D_{4}}$ components of Figure $5(\mathrm{~b}, \mathrm{c})$ are equivalent by a diagram automorphism, say $F_{0}$. The $\widehat{C_{3}}$ components of Figure $5(\mathrm{~b}, \mathrm{c})$ are equivalent by

$$
F_{y} F_{\beta_{3}} F_{y}\left(y, \beta_{2}\right)=F_{y} F_{\beta_{3}}\left(y, \beta_{3}, \beta_{2}\right)=F_{y}\left(y, \beta_{3}\right)=(y) .
$$

So $\mathcal{F}=\left(F_{0}, F_{y}, F_{\beta_{3}}, F_{y}\right)$ transforms Figure $5($ b) to Figure 5(c). If we apply $\mathcal{F}$ to Figure 4(c), it involves a hidden diagram symmetry $F_{0}$ and pushing a hidden button $y$, both of which do not appear in D. By Corollary 1.2, Figure 4(c,d) represent a real form $\widehat{\mathfrak{s}}_{-}$of $L$, where $\mathfrak{s}$ is any real form $\stackrel{\circ}{L}$.

Let us find the members of $\mathcal{V}(\mathrm{D})$ which represent Hermitian real forms of $L$. We minimize the circled vertices by [17, Thm. 6.96] and $F_{\overline{1}}$ of (3.8), and consider only

$$
\left\{(X, Y) ; X=\emptyset,\left(\alpha_{1}\right),\left(\alpha_{3}\right),\left(\alpha_{4}\right) \text { and } Y=\emptyset,\left(\beta_{1}\right),\left(\beta_{2}\right)\right\} \subset \mathcal{V}(\mathrm{D}) \text {. }
$$

Suppose that a Hermitian real form $\mathfrak{g}$ is represented by $c$ in (5.4). By Definition 1.3, $\delta$ is compact and $\mathfrak{g} \cap \stackrel{\circ}{L}$ is Hermitian. They are equivalent to

(a) $\chi(c)=1$,

(b) each connected component of $\widehat{c}_{\overline{0}}$ is equivalent to $\emptyset$ or $(u, v)$ with $b_{u}=b_{v}=1[6,(2.2)(\mathrm{a})]$.

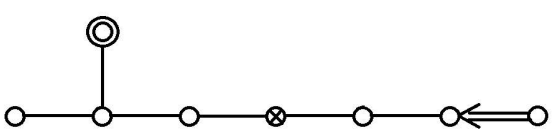

(a)

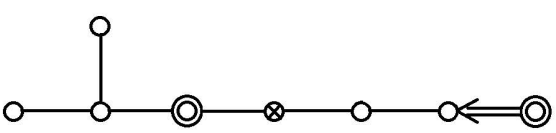

(c)

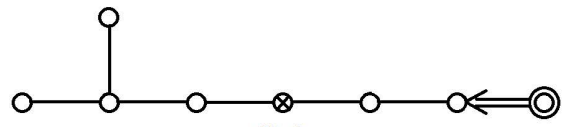

(b)

Figure 6: Hermitian real forms of $D(4,3)$. 
We ignore the diagram with no circled vertex, and select the members of (5.4) which satisfy (5.5). For example, consider Figure 6(a), where $c=\left(\alpha_{1}\right)$. It satisfies $(5.5)$ (a) because $\chi(c)=(-1)^{a_{\alpha_{1}}}(i)^{a_{\gamma}}=(-1)^{1}(i)^{2}=1$. It also satisfies (5.5)(b) because $\widehat{c}_{\overline{0}}=\left(\alpha_{1}, x\right)$ and $b_{\alpha_{1}}=b_{x}=1$. So Figure 6(a) represents a Hermitian real form of $L$. There are two other members of (5.4) which satisfy $(5.5)$, given by Figure $6(\mathrm{~b}, \mathrm{c})$. Figure 6 represents all the Hermitian real forms of $D(4,3)$. They are given by $\widehat{\mathfrak{s}}_{+}$ with the following $\mathfrak{s}_{\overline{0}}$ :
(a) $\mathfrak{s o}(2,6)+\mathfrak{s p}(3)$,
(b) $\mathfrak{s o}(8)+\mathfrak{s p}(3, \mathbb{R})$,
(c) $\mathfrak{s o}(6,2)+\mathfrak{s p}(3, \mathbb{R})$. 


\section{Appendix: Inner automorphisms}

The purpose of this appendix is to give a proof of the following theorem, that is used in the proofs of Proposition 2.4 and Theorem 3.3.

Theorem 6.1. Let $L$ be an affine non-twisted Lie superalgebra. Any inner automorphism of $L_{\overline{0}}$ can be extended to an inner automorphism of $L$.

Before we are able to give the proof of such result, we need to define the notion of inner automorphism in this context. To this aim, we introduce loop groups together with some terminology from algebraic geometry. Our treatment is based on the work by Kumar [18, Ch. XIII].

Let $\stackrel{\circ}{L}_{\overline{0}}$ the even part of a finite dimensional complex contragredient Lie superalgebra with $L_{\overline{0}}$ as its affinization. Let $\stackrel{G}{\overline{0}}_{\overline{0}}$ be the simply connected complex algebraic group associated with $\stackrel{\circ}{\overline{0}}_{\overline{0}}, \operatorname{Lie}\left(\stackrel{\circ}{\overline{0}}_{\overline{0}}\right)=\stackrel{\circ}{\bar{L}}_{\overline{0}}$. Here $\stackrel{\circ}{\overline{0}}_{\overline{0}}$ is a matrix group, so $\stackrel{\circ}{\overline{0}}_{\overline{0}} \subset \mathrm{GL}_{N}(\mathbb{C})$ for a suitable $N$. As for any algebraic group, we can consider $\dot{G}_{\overline{0}}(R)$, the $R$ points of $\dot{G}_{\overline{0}}$ for a complex algebra $R$. By definition $\dot{G}_{\overline{0}}(R)$ is the group of all the algebra morphisms $\mathbb{C}\left[\stackrel{\circ}{\bar{G}}_{\overline{0}}\right] \longrightarrow R$, where $\mathbb{C}\left[\stackrel{\circ}{\overline{0}}_{\overline{0}}\right]$ is the coordinate ring of the algebraic group $\dot{G}_{\overline{0}}$. Notice that $\dot{G}_{\overline{0}}(R)$ is a subgroup of $\mathrm{GL}_{N}(R)$.

Definition 6.2. We define loop group associated with $\stackrel{\circ}{L}$ :

$$
\mathcal{L}\left(\stackrel{\circ}{\overline{0}}_{\overline{0}}\right)=\stackrel{\circ}{\bar{G}}_{\overline{0}}(K), \quad K:=\mathbb{C}[[t]]\left[t^{-1}\right]
$$

We are actually interested in a larger group, since it is clear that $\mathcal{L}\left(\dot{G}_{\overline{0}}^{\circ}\right)$, by its very definition, can encode information only about the loop algebra $\mathbb{C}\left[t, t^{-1}\right] \otimes \stackrel{\circ}{\bar{L}}_{\overline{0}}$ inside $L_{\overline{0}}$.

Consider the morphism $\gamma: \mathbb{C}^{\times} \longrightarrow \operatorname{aut}(K), z \mapsto \gamma_{z}$, where $\gamma_{z}(p(t)):=p(z t)$ and $p(t) \in K$. This induces a group morphism, still denoted by $\gamma$, where $\gamma: \mathbb{C}^{\times} \longrightarrow$ $\operatorname{aut}\left(\mathcal{L}\left(\stackrel{\circ}{\overline{0}}_{\overline{0}}\right)\right)$. It allows us to define the group $\overline{\mathcal{L}}\left(\stackrel{\circ}{G}_{\overline{0}}\right)$ as the semidirect product

$$
\overline{\mathcal{L}}\left(\stackrel{\circ}{G}_{\overline{0}}\right):=\mathbb{C}^{\times} \ltimes \mathcal{L}\left(\stackrel{\circ}{\overline{0}}_{\overline{0}}\right) .
$$

We denote by $d_{z}$ the element $(z, 1)$, which heuristically we interpret as the exponential of the derivation $d$ in $L_{\overline{0}}$.

We wish to define the adjoint action of $\overline{\mathcal{L}}\left(\stackrel{G}{\overline{0}}_{\overline{0}}\right)$ on the superalgebra $L$ and relate it to the works by Batra, Messaoud and Rousseau [2], [20]. This will lead us to the definition of inner automorphisms of $L$ and to the proof of Theorem 6.1.

The algebraic group $\stackrel{\circ}{\overline{0}}_{\overline{0}}$ acts via the adjoint action on its Lie algebra $\stackrel{\circ}{L}_{\overline{0}}$. Such action is naturally extended to the (finite dimensional) contragredient complex superalgebra $\stackrel{\circ}{L}$ (see for example [3, Ch. 7]). In the functor of points notation, we can write for any algebra $R$ :

$$
\operatorname{Ad}_{R}(g)(x)=g x g^{-1}, \quad g \in \stackrel{\circ}{G}_{\overline{0}}(R), x \in R \otimes \stackrel{\circ}{L} .
$$


We remark that, by the results in [3, Ch. 7], in principle, we can define $\operatorname{Ad}_{R}(g)(x)$ only for $x \in(R \otimes \stackrel{\circ}{L})_{\overline{0}}$. However, since $\operatorname{Ad}_{R}$ is a linear action, we can immediately extend the definition to $x \in R \otimes \stackrel{\circ}{L}$.

In analogy with [18, Ch. XIII, 13.2], we give the following definition.

Definition 6.3. Let the notation be as above. We define the adjoint map:

$$
\operatorname{Ad}: \overline{\mathcal{L}}\left(\stackrel{\circ}{G}_{\overline{0}}\right) \longrightarrow \operatorname{aut}(L)
$$

as follows. Let $x \in K \otimes \stackrel{\circ}{L}, \lambda, \mu \in \mathbb{C}, z \in \mathbb{C}^{\times}, g \in \mathcal{L}\left(\dot{G}_{\overline{0}}\right)$ (that is $g \in \dot{G}_{\overline{0}}(K)$ ), we define

$$
\begin{aligned}
\operatorname{Ad}(g)(x+\lambda c+\mu d) & :=\operatorname{Ad}_{K}(g)(x)-\mu t\left(\frac{d g}{d t}\right) g^{-1} \\
& +\left(\lambda-\operatorname{res} B\left(g^{-1} \frac{d g}{d t}, x-\frac{1}{2} \mu t g^{-1} \frac{d g}{d t}\right)\right) c+\mu d \\
\operatorname{Ad}\left(d_{z}\right)(x+\lambda c+\mu d) & :=\gamma_{z}(x)+\lambda c+\mu d .
\end{aligned}
$$

Notice that for $L=L_{\overline{0}}$, this is the formula (3) in [18, Sec. 13.2].

Proposition 6.4. Ad is a representation of the group $\overline{\mathcal{L}}\left(\stackrel{\circ}{G}_{\overline{0}}\right)$ on the Lie superalgebra $L$.

Proof. The case of $L=L_{\overline{0}}$ is discussed in [18, 13.2.3]. First notice $\operatorname{Ad}(g) \in \operatorname{aut}(L)$. In fact $\operatorname{Ad}(g)(x+\lambda c+\mu d) \in L_{\overline{0}}$ for $x$ even [18, 13.2.3], and $\operatorname{Ad}(g)(x) \in L_{\overline{1}}$ for $x$ odd, because $\operatorname{Ad}_{K}(g)(x) \in K \otimes \stackrel{\circ}{L}_{1}\left[3\right.$, Ch. 7]. Furthermore $\operatorname{Ad}_{K}(g)$ is invertible, because its restriction to $L_{\overline{0}}$ is invertible.

It remains to check $\operatorname{Ad}(g)$ and $\operatorname{Ad}\left(d_{z}\right)$ preserve the bracket of two odd elements, since the case of even elements is covered in $[18,13.2 .3]$, and the case of an even and an odd element is the same. Since $\lambda c+\mu d$ is even, we consider only $x, y \in K \otimes \stackrel{\circ}{L}$,

$$
\operatorname{Ad}(g)[x, y]=\operatorname{Ad}_{K}(g)[x, y]=g(x y+y x) g^{-1}=[\operatorname{Ad}(g) x, \operatorname{Ad}(g) y] .
$$

The check for $\operatorname{Ad}\left(d_{z}\right)$ is also immediate.

We now wish to relate the treatment of Kumar to the results in [20] and [2], then generalize them to the super setting. Proposition 6.4 leads to the following definition.

Definition 6.5. We define an inner automorphism of $L$ as an element of $\operatorname{Ad}(\overline{\mathcal{L}}(\stackrel{\circ}{G}))$, and we call $\operatorname{Ad}(\overline{\mathcal{L}}(\stackrel{\circ}{G}))$ the group of the inner automorphisms of $L$. We denote it by $\operatorname{int}(L)$.

The next observation establishes a bridge between Definition 6.5 and the one in [2], appearing in the results we are using. 
Observation 6.6. For $L=L_{\overline{0}}$, Definition 6.5 agrees with the definition given in [20, Sec. 1.4], which is used in [2]. In fact, [20, Sec. 1.2] introduces the group $G$ generated

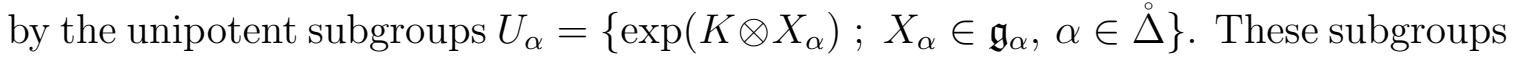
are also examined in [18, Sec. 13.2, Prop. 13.2.4], with a more detailed treatment. Then, in [20, Sec. 1.4], the authors define the semidirect product $\widetilde{H} \ltimes G$ between the torus $\widetilde{H}=\exp (\mathfrak{h})$ and $G$. Notice that this group is precisely $\mathcal{G}$, the Kac-Moody group introduced in $[18$, Secs. 6.1, 13.2]. In fact both groups are generated by the same elements. The group $\mathcal{G}$ is closely related with the group $\overline{\mathcal{L}}(\stackrel{\circ}{G})$ we have used for Definition 6.3. In fact by [18, Thm. 13.2.8], we have $\overline{\mathcal{L}}(\stackrel{\circ}{G})=\mathcal{G} / Z(\mathcal{G})$. Going back to [20], we see that in there the inner automorphism group $\operatorname{int}(L)$ is defined as $\operatorname{Ad}(\widetilde{H} \ltimes G)$. In such action the center acts trivially, hence their definition of inner automorphisms of $L$ agrees with our Definition 6.5.

Proof of Theorem 6.1.

Any inner automorphism of $L_{\overline{0}}$, by Definition 6.5 and Observation 6.6, is evidently the restriction of an inner automorphism of $L$.

Acknowledgements. We would like to thank Prof. V. Serganova for helpful comments. M. K. Chuah is supported by a grant from Gnsaga, Indam and he would like to thank the Department of Mathematics at the University of Bologna for the wonderful hospitality while this work was done. 


\section{References}

[1] A. Alldridge, Fréchet globalisations of Harish-Chandra supermodules, arXiv:1403.4055, 2014.

[2] P. Batra, Invariants of real forms of affine Kac-Moody Lie algebras, J. Algebra 223 (2000), 208-236.

[3] C. Carmeli, L. Caston and R. Fioresi, Mathematical Foundation of Supersymmetry, with an appendix with I. Dimitrov, EMS Ser. Lect. Math., European Math. Soc., Zurich, 2011.

[4] C. Carmeli, R. Fioresi and V. S. Varadarajan, Highest weight Harish-Chandra supermodules and their geometric realizations, Transf. Groups, 2018.

[5] M. K. Chuah, Finite order automorphisms on contragredient Lie superalgebras, J. Algebra 351 (2012), 138-159.

[6] M. K. Chuah, Finite order automorphisms on real simple Lie algebras, Trans. Amer. Math. Soc. 364 (2012), 3715-3749.

[7] M. K. Chuah, Cartan automorphisms and Vogan superdiagrams, Math. Z. 273 (2013), 793-800.

[8] M. K. Chuah and R. Fioresi, Hermitian real forms of contragredient Lie superalgebras, J. Algebra 437 (2015), 161-176.

[9] M. K. Chuah and R. Fioresi, Admissible positive systems of affine non-twisted Kac-Moody Lie algebras J. Algebra 453 (2016), 561-577.

[10] M. K. Chuah and R. Fioresi, Admissible positive systems of affine Kac-Moody Lie algebras: the twisted cases J. Algebra 469 (2017), 390-401.

[11] M. K. Chuah and C. C. Hu, Equivalence classes of Vogan diagrams, J. Algebra 279 (2004), 22-37.

[12] M. K. Chuah and C. C. Hu, Extended Vogan diagrams, J. Algebra 301 (2006), $112-147$.

[13] R. Fioresi, Compact forms of complex Lie supergroups, J. Pure Appl. Alg. 218 (2014), 228-236.

[14] Harish-Chandra, Representations of semisimple Lie groups IV, Amer. J. Math. 77 (1955), 743-777. 
[15] V. G. Kac, Lie superalgebras, Adv. Math. 26 (1977), 8-96.

[16] V. G. Kac, Infinite dimensional Lie algebras, 3rd. ed., Cambridge University Press, Cambridge 1990.

[17] A. W. Knapp, Lie Groups beyond an Introduction, 2nd. ed., Progr. Math. vol. 140, Birkhäuser, Boston 2002.

[18] S. Kumar, Kac-Moody Groups, their Flag Varieties and Representation Theory, Birkhäuser, 2002.

[19] F. Levstein, A classification of involutive automorphisms of an affine Kac-Moody Lie algebra, J. Algebra 114 (1988), 489-518.

[20] H. B. Messaoud and G. Rousseau, Classification des formes réelles presque compactes des algèbres de Kac-Moody affines, J. Algebra 267 (2003), 443-513.

[21] I. M. Musson, Lie superalgebras and Enveloping Algebras, Graduate Studies in Mathematics, vol. 131, American Mathematical Society, 2012. 